\title{
Experimental studies and Monte Carlo simulations for beam loss monitors
}

\author{
Tao Yang $\odot,{ }^{1,2,3, *}$ Jianmin Tian $\odot,{ }^{1,2}$ Lei Zeng, ${ }^{1,2}$ Taoguang Xu๑®, ${ }^{1,2}$ Weiling Huang, ${ }^{1,2}$ Jilei Sun, ${ }^{1,2}$ \\ Fang Li ${ }^{1,2}$ Ruiyang Qiu, ${ }^{1,2}$ Zhihong Xu, ${ }^{1,2}$ Ming Meng, ${ }^{1,2}$ Anxin Wang, ${ }^{1,2}$ Peng Li ${ }^{1,2}$ \\ Mengyu Liu, ${ }^{1,2,3}$ Xiaojun Nie, ${ }^{1,2}$ Jianrong Zhou, ${ }^{1,2,4}$ Zhijia Sun, ${ }^{1,2,3,4}$ and Qun Ouyang ${ }^{1,3,4}$ \\ ${ }^{1}$ Institute of High Energy Physics, Chinese Academy of Sciences (CAS), Beijing 100049, China \\ ${ }^{2}$ Spallation Neutron Source Science Center, Dongguan 523803, China \\ ${ }^{3}$ University of Chinese Academy of Sciences, Beijing 100049, China \\ ${ }^{4}$ State Key Laboratory of Particle Detection and Electronics, Beijing 100049, China
}

(Received 13 March 2020; revised 24 November 2020; accepted 17 February 2021; published 16 March 2021)

\begin{abstract}
Beam loss detection is essential for the machine protection and the fine-tuning of the accelerator to reduce the induced radioactivity. Monte Carlo simulations are also vital for the choice of beam loss monitor (BLM) type, for predicting and understanding the BLM response to beam losses. At the China Spallation Neutron Source (CSNS), the cylindrical ionization chamber (IC) filled with $\mathrm{Ar} / \mathrm{N}_{2}$ gas mixture is the main type of the BLM to detect the beam losses. This paper presents the detailed beam loss experiments and FLUKA simulations for the CSNS BLM system. It includes the response functions of the BLM detectors by taking into account the contributions of different secondary particles to the energy deposition in the sensitive volume of the BLM. Dedicated experiments were compared with FLUKA simulations. It was found that in the low energy section of the linac the usual ion chamber based BLMs are not sensitive enough. A $\mathrm{BLM}$ based on $\mathrm{BF}_{3}$ enclosed by a high-density polyethylene (PE) moderator is effective to detect the beam losses through detecting thermal neutrons. Its signal is about 3 orders of magnitude higher than that of $\mathrm{Ar} / \mathrm{N}_{2}$ BLM for a beam energy of $15 \mathrm{MeV}$. The simulations provide the results of the intrinsic delay time for the $\mathrm{BF}_{3}$ monitor and the thickness optimization for $\mathrm{PE}$ to reduce the delay. Finally, the simulated spatial resolution of loss location by detection of neutrons is also evaluated for a beam energy of $15 \mathrm{MeV}$, which presents a resolution of $\sim 2 \mathrm{~m}$ in our experimental configuration.
\end{abstract}

DOI: 10.1103/PhysRevAccelBeams.24.032804

\section{INTRODUCTION}

Neutron beams are created at the China Spallation Neutron Source (CSNS) through the spallation reaction of $1.6-\mathrm{GeV}$ proton beams striking on the tungsten target, and then widely utilized in various studies of material science and bioscience [1]. Figure 1 depicts the schematic layout of the accelerator, which is mainly comprised of a $50-\mathrm{keV} \mathrm{H}^{-}$ion source, a 3-MeV radio frequency quadrupole (RFQ) accelerator operating at a frequency of $324 \mathrm{MHz}$, an 80-MeV drift tube linac (DTL), and a 1.6-GeV proton rapid cycle synchrotron (RCS). The peak current is about $15 \mathrm{~mA}$ with a repetition rate of $25 \mathrm{~Hz}$ in the linac, and the macropulse duty factor up to $1.25 \%$. After

\footnotetext{
*Corresponding author. yangt@ihep.ac.cn

Present address: No. 1, Zhongziyuan Road, Dalang, Dongguan 523803, China.

Published by the American Physical Society under the terms of the Creative Commons Attribution 4.0 International license. Further distribution of this work must maintain attribution to the author(s) and the published article's title, journal citation, and DOI.
}

chopping, the micropulse has a length of about $415 \mathrm{~ns}$ with a repetition rate of $1 \mathrm{MHz}$. The RCS is generally operated in the double-bunch mode per pulse. Each pulse contains $1.56 \times 10^{13}$ protons, and each of the bunch in it is $\sim 500 \mathrm{~ns}$ in length and the time interval between them is also $\sim 500$ ns just after the injection. Then the two bunches are accelerated and compressed. At the time of bunch extraction, the length is rendered to $\sim 80 \mathrm{~ns}$ and the time interval becomes $\sim 330 \mathrm{~ns}$. The main machine parameters are listed in Table I. CSNS had generated the neutron beam in August 2017 and now it is in the phase of upgrading the beam power to $100 \mathrm{~kW}$.

During the running and commissioning of the accelerator facility, the problem caused by beam losses is one of the most important since beam loss might damage the accelerator components severely due to the high energy deposition $[2,3]$, and it may produce undesired radioactivation $[3,4]$. The worst case is the uncontrolled accident loss; it may induce destructive damage to accelerator components which resulted from thermal shock [5].

There exist several types of beam loss monitors (BLMs) based on different detection principles such as ionization (e.g., IC, PIN diodes, and diamonds), scintillation (photomultipliers with scintillators), secondary emission, and 


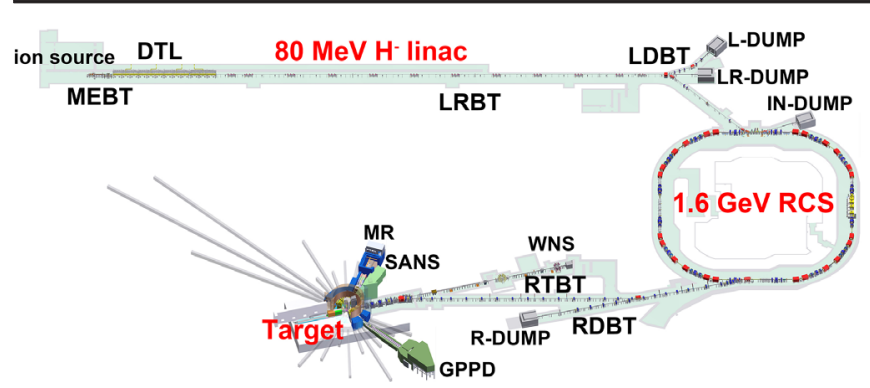

FIG. 1. The schematic layout of accelerator and target in CSNS.

Cherenkov light [3]. CERN's Large Hadron Collider (LHC) BLM system mainly uses the parallel plate IC and secondary emission monitors (SEM); both monitors are filled with nitrogen at a pressure of 1.1 bar [6]. The Japan Proton Accelerator Research Complex (J-PARC) BLM system is composed of the coaxial cable air-filled IC, scintillator with photomultipliers, and proportional counters [7]. The facility for rare isotope beams (FRIB) has developed the loss monitor ring (LMR) type BLM for monitoring the beam loss in the low-energy heavy-ion accelerators [8]. The Stanford Linear Accelerator Center (SLAC) adopts the Panofsky long ionization chamber to detect the beam loss, whose cable length is $3 \mathrm{~km}$ long. The loss points can be determined with a resolution of several meters by using the fast gas $\mathrm{Ar} / \mathrm{CF}_{4}$ [9]. Distributed BLMs based on Cherenkov effect in an optical fiber have been widely used in light sources, such as Deutsches ElektronenSynchrotron (DESY) and SPring-8 Angstrom Compact free electron laser (SACLA) due to the ultrafast response (less than $1 \mathrm{~ns})[10,11]$. However, for hadron machines, currently IC is still the main BLM type (SNS, LHC, RHIC, ESS, etc.) [6,12-14] due to its robustness to radiation damage, large dynamic range, little maintenance, and ease for calibration.

In practice, a single BLM type is not capable of satisfying a high dynamic range of beam loss detection. The beam loss monitoring system in the CSNS mainly applies two types of monitors based on IC and plasticscintillator respectively. There are 168 IC-type BLMs along the beam line in the CSNS, mostly filled with $\mathrm{Ar} / \mathrm{N}_{2}$ mixture gas, several filled with $\mathrm{Xe}$ or $\mathrm{BF}_{3}$ gas, and there are

TABLE I. The basic design parameters of CSNS.

\begin{tabular}{lc}
\hline \hline Design parameter & Value \\
\hline Beam power $(\mathrm{kW})$ & 100 \\
Linac energy $(\mathrm{MeV})$ & 80 \\
Beam current in the linac $(\mathrm{mA})$ & 15 \\
Extraction energy $(\mathrm{GeV})$ & 1.6 \\
Proton per pulse & $1.56 \times 10^{13}$ \\
Repetition rate $(\mathrm{Hz})$ & 25 \\
Linac rf frequency $(\mathrm{MHz})$ & 324 \\
Target material & Tungsten \\
\hline \hline
\end{tabular}

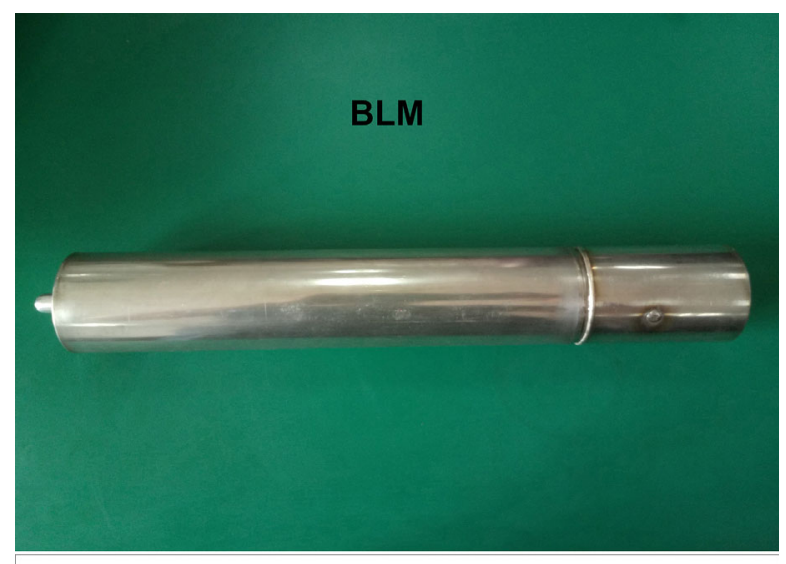

\section{BLM geometric model}

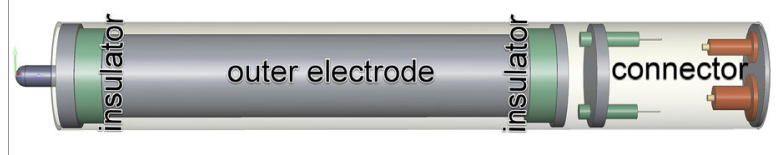

FIG. 2. The BLM used in CSNS and its geometric model implemented in FLUKA simulations.

also 15 scintillator-based BLMs. Plastic scintillator detectors have the advantages of high sensitivity to radiation and ultrafast response, thus this type of BLM is utilized together with the IC-type BLMs to fine-tune the accelerator. This paper mainly focuses on the IC-type BLMs; for convenience, the term BLM(s) mentioned below is only referred to as the IC-type BLM(s).

The CSNS BLM is cylindrical, with its appearance and the geometric model for Monte Carlo simulations shown in Fig. 2. The detailed geometric and operation parameters are listed in Table II. The sensitive volume is the tubelike region enclosed by the outer and inner electrode with a length of $17.4 \mathrm{~cm}$. Energy deposition only in this region contributes to the BLM signal. The outer radius of the BLM is $2.5 \mathrm{~cm}$, filled with a mixture of argon and nitrogen (70:30 by volume) with the pressure of $1 \mathrm{~atm}$. The standard bias voltage on the outer electrode in normal operation is set to be $-2100 \mathrm{~V}$. The electrons are collected on the inner electrode. All electrodes and coverages of our BLMs are made of stainless steel, and the insulators are made of ceramics $\left(\mathrm{Al}_{2} \mathrm{O}_{3}\right)$. The BLMs are operated in the ionization chamber region, where the electric field should be sufficiently strong to separate the ionization products and prevent recombination, however, it should be well below the gas amplification threshold [15]. Figure 3 shows several BLMs installed nearby different components along the beam line. At least one BLM should be mounted at every 


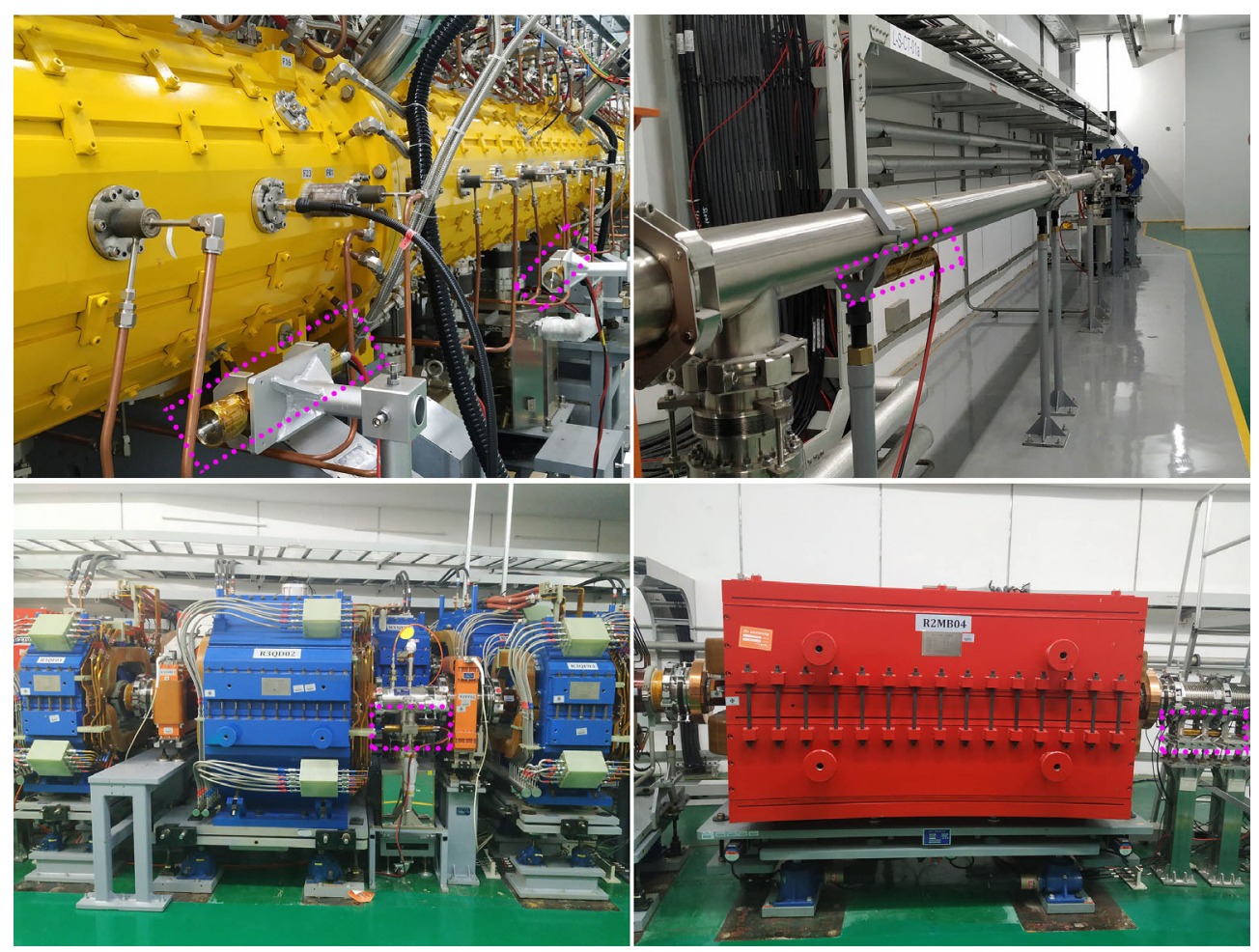

FIG. 3. Several BLMs installed along the CSNS beam line.

quadrupole where the beam loss has a high probability [16]. The best mounting position can be found by simulations taking account of the beam energy, particle type, magnetic strength, and length of the quadrupole.

Beam losses can generally be categorized into two groups: the slow loss (regular loss) and fast loss (irregular loss) $[3,16]$. The slow losses can be caused by various effects: Touschek effect, collisions, transversal and longitudinal diffusion, residual gas scattering, beam instability, etc. The slow losses occur continuously during the accelerator running and are typically not avoidable due to the intrinsic characteristics of beam transport. The fast losses

TABLE II. The basic design and operation parameters of the CSNS BLM.

\begin{tabular}{lc}
\hline \hline Design parameter & Value \\
\hline Length $(\mathrm{cm})$ & 32.45 \\
Length of sensitive volume $(\mathrm{cm})$ & 17.4 \\
Diameter $(\mathrm{cm})$ & 5.0 \\
Radius of outer electrode $(\mathrm{cm})$ & 1.925 \\
Radius of inner electrode $(\mathrm{cm})$ & 1.27 \\
Electrode thickness $(\mathrm{cm})$ & 0.015 \\
Wall thickness $(\mathrm{cm})$ & 0.1 \\
Filled gas & $70 \% \mathrm{Ar}+30 \% \mathrm{~N}_{2}$ \\
Gas pressure $($ atm) & 1.0 \\
Electrode/wall material & Stainless steel \\
Standard bias voltage $(\mathrm{V})$ & 2100 \\
Gas leak rate $(\mathrm{Pa} \cdot \mathrm{L} / \mathrm{s})$ & $<1 \times 10^{-8}$ \\
\hline \hline
\end{tabular}

are often caused by the beam misalignment or some operation failures, such as the fault of the rf or magnet power supply system, which may cause serious damage to accelerator components. In general, the beam loss monitoring system requires a high dynamic range to detect the two types of losses. The input current ranges from $200 \mathrm{pA}$ to $20 \mu \mathrm{A}$ (dynamic range: $10^{5}$ ) for the electronics of BLMs used in CSNS. The signal to noise ratio at $200 \mathrm{pA}$ is determined by the noise level of the electronics system and is about $9 \mathrm{~dB}$ when the BLMs are installed in the accelerator tunnel. According to the facility operation experiences in CSNS, the most frequent causes of regular beam loss are beam instability, residual gas scattering, and space charge effects, while the most frequent cause of irregular loss is the failure of the magnet power supply

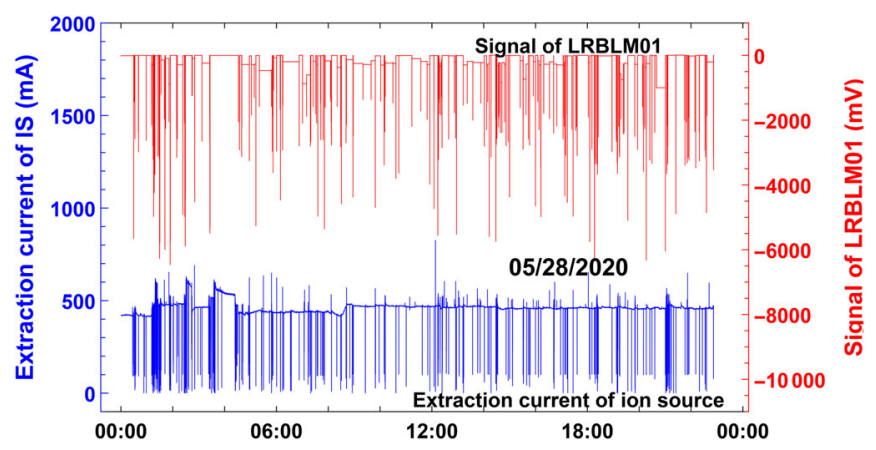

FIG. 4. A BLM signal versus the synchronous extractioncurrent signal of the ion source. 


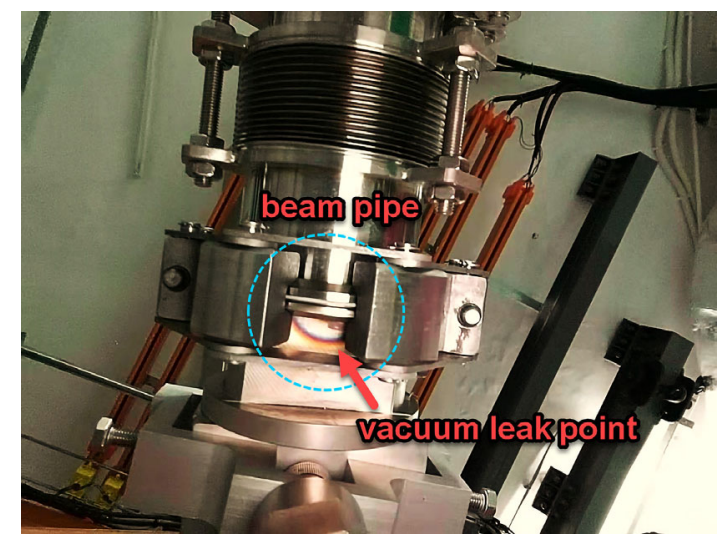

FIG. 5. A beam loss event leading to a vacuum leak accident in CSNS.

system. Up to now, the observed beam instability is most likely originated from the sparking of the ion source and RFQ. Figure 4 shows a BLM signal versus the synchronous extraction-current signal of ion source, which apparently reveals the time relevance of the BLM signal on the sparking. Figure 5 depicts an irregular beam loss event in CSNS, which caused a partial melting in the beam pipe in the linac to ring beam transport line (LRBT) section where the beam energy is $\sim 80 \mathrm{MeV}$ and the pulsed peak current is $\sim 15 \mathrm{~mA}$. This irregular beam loss eventually led to a vacuum leak accident, which resulted in an accelerator shutdown to locate the leak point. The postanalysis revealed that this accident was caused by the wrong setting of the switch magnet in the LRBT part. The misalignment of the beam consequently rendered a high-intensity bombardment of beam particles, damaging the beam pipe.

Beam loss detection is essential for the two classes of losses, and BLMs are one of the most crucial components of the machine protection system (MPS) along the entire accelerator beam line [17]. The protection mechanism is as follows: first, a protection threshold of the BLM signal is set, which is adjusted during the machine tuning; then MPS can request the beam dump or emergency extraction if the value of signal of one loss event exceeds the preset threshold. The MPS strategy in CSNS is to switch off the timing system of the extraction power supply of the ion source to shut down the beam. In practice, this overthreshold signal will be further processed and then sent to the MPS, which gives a comprehensive judgment and an action with a well-designed electronics and control system. At present, the machine protection strategy from the BLM system in CSNS is to judge the occurrence frequency of the beam loss overthreshold signal. A casual overthreshold event will shut down the beam for a second and then the beam is allowed for automatic recovery to normal operation, meanwhile, the MPS will send an alert. However, the MPS will trigger the beam interlock system to stop the beam permanently if the beam loss signal exceeds the threshold more than 8 times in ten seconds in the linac or 2 times in five seconds in the RCS, which will require manual recovery. The occurrence frequency triggering the interlock system can be adjusted according to the actual situations. In consequence, the beam loss detection mainly has two purposes: (1) trigger the MPS to shut down the beam or extract it to the dump if the beam loss signal exceeds the threshold, (2) fine-tune the accelerator to decrease the beam loss and the consequent induced radioactivity as low as possible.

The Monte Carlo program FLUKA [18-20] is employed to perform the simulations, such as $\gamma$-ray irradiation, the response functions of BLMs, beam loss responses in several assumed loss scenarios along the entire beam line, etc. The contributions from the secondary particles to signal are analyzed using the two-steps method [21]. Based on different loss rates, the macroscopic current signal of BLMs is simulated, and influences of the space charge effect on the collection efficiency are also evaluated. Through detailed Monte Carlo simulations, we can predict the signal levels in an assumed loss scenario, and then we could know whether the BLM and the electronics are suitable. Extensive experimental studies are also performed to validate FLUKA simulations in this paper.

Detection of beam loss in the low energy section (e.g., $<20 \mathrm{MeV}$ in CSNS) of a proton accelerator is a difficult task since the thick tank ( $\sim 4.2 \mathrm{~cm}$ in CSNS) of DTL will introduce a considerable shielding effect for the beam-loss induced secondary particles, which will significantly reduce the signal at the BLM locations. Fortunately, it is an effective way to detect the beam loss in the low energy section by detecting the nuclear reaction products of thermal neutrons interacting with high cross-section nuclides like ${ }^{3} \mathrm{He}$ and ${ }^{10} \mathrm{~B}$ [15]. In our experiments, we manage to detect the beam loss signal by using the $\mathrm{BF}_{3}$ BLM enclosed with a $7.5-\mathrm{cm}$ thick PE moderator to slow down secondary neutrons. The simulation result agrees well with the experimental value, both of which show the signal of $\mathrm{BF}_{3} \mathrm{BLM}$ is about 3 orders of magnitude higher than that of the commonly used $\mathrm{Ar} / \mathrm{N}_{2}$ BLMs in CSNS. The spatial resolution of the beam loss point is calculated through the distribution of secondary neutrons.

\section{SIMULATION AND EXPERIMENTAL STUDIES FOR CSNS BLMS}

\section{A. Particle-matter interactions}

BLMs detect the secondary particles of the lost particles interacting with the accelerator components. The secondary radiation field along a high energy proton accelerator is often composited by the following particles: $\gamma$ rays, neutrons, protons, electrons, positrons, pions, and muons [22]. The detection process of beam loss can be simply divided into two steps: the lost particles of the primary beam interact with the accelerator components, then the produced secondary particles arrive at the BLMs location 

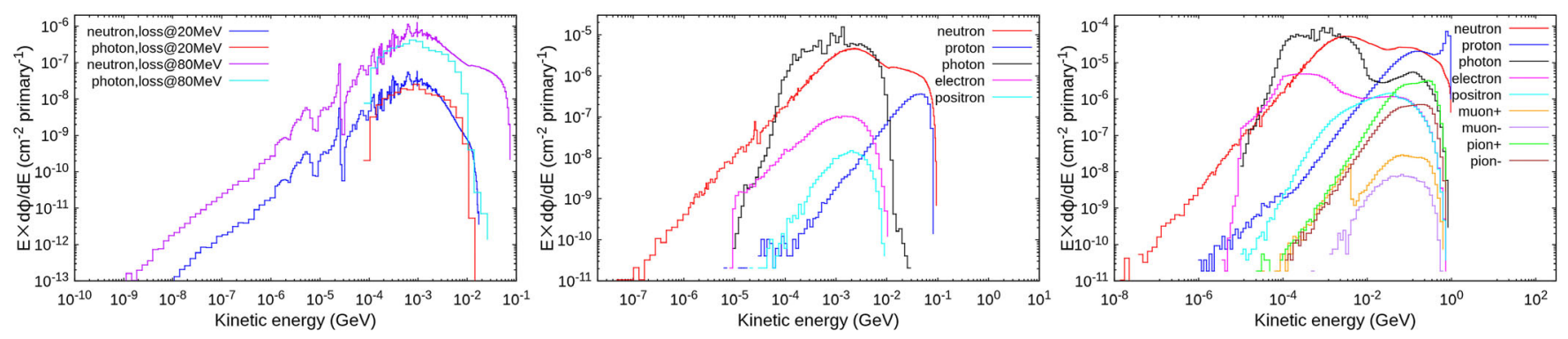

FIG. 6. Secondary particle fluence spectra outside of the DTL tank for beam loss at the energy of 20 and $80 \mathrm{MeV}$ (left) and spectra outside of the RCS beam pipe at the energy of $100 \mathrm{MeV}$ (middle) and $1.0 \mathrm{GeV}$ (right).

and dissipate energy in the sensitive volume to generate an input signal of electronics for further processing.

In a proton accelerator, the lost proton penetrating a material will lose its energy through several different processes depending on its energy. In general, protons with lower energies will lose energy mainly via the electromagnetic interaction with atomic electrons. More specifically, protons will undergo the ionization process if the transfer energy exceeds the binding energy of a shell electron, otherwise, they will release the energy by the excitation of atoms. The proton can be deflected from its original trajectory by the elastic Coulomb interactions with nuclei. Nuclear reactions may also occur for proton energy high enough to overcome the Coulomb barrier of target nuclei [23]. With the increase of proton energy, hadronic and electromagnetic cascades will start. The production threshold for the $\left(p, \pi^{ \pm}\right)$reaction can be calculated as follows [23]:

$$
T_{\mathrm{th}}=2 m_{\pi} c^{2}\left(1+m_{\pi} / m_{p}\right),
$$

where $T_{\text {th }}$ is the threshold kinetic energy of the proton, $m_{\pi}$ and $m_{p}$ are respectively the rest mass of pion and proton. $T_{\text {th }}$ is $\sim 290 \mathrm{MeV}$ by substituting the mass values. It is worth noting that Eq. (1) supposes the proton interacts with a free nucleon, for a nucleon bound in a nucleus, the threshold is somewhat lower. The produced hadrons will have further inelastic nuclear collisions (nucleon-nucleon reactions, pion-nucleon reactions) and result in a hadronic cascade. The decay products of $\pi^{0}$ particles are $\gamma$ pairs, which can initiate the electromagnetic showers. The charged pions and other hadrons will also have a possibility to decay and produce muons. The secondary charged particles will lose energy mainly through the ionization and excitation process. High-energy electrons also deposit energy by the bremsstrahlung process. The energy loss process of positrons is similar to electrons due to almost the same mass and charge; the main difference is the positron annihilation [15]. Photons and neutrons are neutral particles that cannot directly ionize the medium; they deposit energy mainly through generating secondary charged particles. Photoelectric effect, Compton effect, and electron-positron pair production are the main processes for photons to generate secondary electrons. High-energy photons can also induce photonuclear reactions. Neutrons generate charged particles via different interactions, e.g., elastic/ inelastic scattering, radiative capture, fission reactions, and spallation reactions.

Figure 6 shows the secondary particle spectra outside of the DTL tank for beam loss at the energy of 20 and $80 \mathrm{MeV}$ in the CSNS linac, and the spectra outside of the RCS beam pipe at the energy of $100 \mathrm{MeV}$ and $1.0 \mathrm{GeV}$. The detailed loss scenarios are presented in Sec. II F (scenarios I and II). For beam loss at DTL, we only record the neutral particles neutron and photon due to the shielding effect of the tank, especially for charged particles. In general, the neutron fluence is higher than that of the photon (fluence difference enlarges at $80 \mathrm{MeV}$ compared with $20 \mathrm{MeV}$ ), and both particles form a peak around $1 \mathrm{MeV}$. The fluences of two particles for $80 \mathrm{MeV}$ are about 1 order of magnitude higher than that for $20 \mathrm{MeV}$. In the case of beam loss on beam pipe at $100 \mathrm{MeV}$, photon and neutron fluence is apparently higher than others. Muons and pions have not been generated yet due to the production threshold, while for beam loss at $1.0 \mathrm{GeV}$, all the main secondary particles are produced. Neutrons dominate the spectrum between 2 to $200 \mathrm{MeV}$, protons dominate between $200 \mathrm{MeV}$ to $1.0 \mathrm{GeV}$, and photons dominate between about $30 \mathrm{keV}$ to $2 \mathrm{MeV}$. Electron fluence is considerable below $\sim 100 \mathrm{MeV}$, and higher than positrons. $\pi^{ \pm}$fluence rises up above $\sim 300 \mathrm{MeV}$ and $\mu^{ \pm}$have the minimum one. The relative variations of particle fluence are related to the particlematter interactions presented at the beginning of this section.

\section{B. Simulation considerations and methods}

Monte Carlo simulations are the essential part for the choice of BLM type, the response of BLM to beam loss, the selection of installation location, and even the BLM orientation. Extensive simulations are performed by FLUKA. It is a multipurpose Monte-Carlo particle transport program for simulations of particle interaction with matter by using accurate physical models that are checked against the massive experimental data. The accurate and 
well-tested models of hadron-nucleus and nucleus-nucleus collisions make FLUKA very suitable in the field of hadron accelerators, such as the detector design and optimization, radiation activation and shielding, etc. Besides, the software Flair as FLUKA GUI is an advanced user-friendly interface, which makes it is much easier to facilitate the editing of FLUKA input files, the modeling of complex geometry, execution of the code, the analysis of data, and visualization of the output files [24]. In our simulations, the PRECISIOn defaults are activated using the DEFAULTS card for detailed simulations. The production and transport thresholds are set to $10^{-3}$ and $10^{-4} \mathrm{MeV}$ respectively for electrons and gammas in the BLM sensitive volume, however, the cutoffs are set to higher values (e.g., $100 \mathrm{keV}$ ) in massive bulk materials like magnet yoke or DTL tank for the consideration of saving computing time. FLUKA offers a rich choice of options for scoring most quantities, however, there exist special cases that the required information cannot be obtained through built-in options, then the use of user routines is necessary in these cases.

The energy deposition of radiation in matter follows the statistical nature. In general, when a radiation particle penetrates a thin solid layer or gas layer, the energy deposition fluctuation is described by the Landau distribution [25]. Figure 7 shows the energy deposition for $1 \mathrm{GeV}$ protons traversing the BLM, and the simulation data fits well with the Landau distribution. Due to the skewness of Landau distribution, the mean value of energy deposition is not equal to the most probable value, and the former one is larger. The mean value of the deposited energy is important for the calculation of BLM responses because the measurement is done by integrating the created charges.

The charge created in the sensitive volume of BLMs is calculated by the following equation [26]:

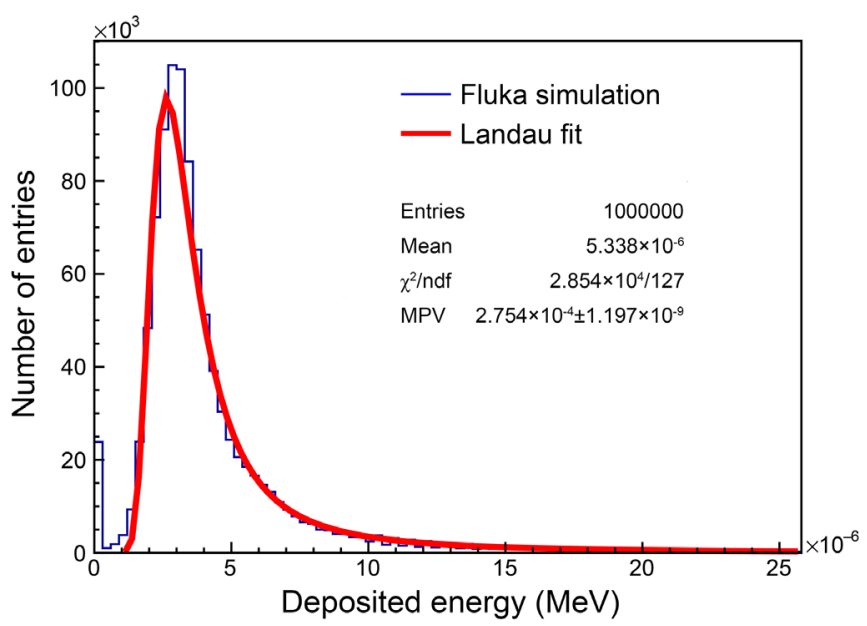

FIG. 7. Landau distribution of energy deposition for $1 \mathrm{GeV}$ protons traversing the CSNS BLM.

$$
Q=\frac{E_{\mathrm{dep}} \cdot e}{W},
$$

where $E_{\mathrm{dep}}$ is the total deposited energy, $e$ the elementary charge, and $W$ value the average energy required to produce an electron-ion pair. For a mixture gas, $W$ value is approximately acquired as follows [27]:

$$
\left(\frac{1}{W}\right)_{\text {mix }} \cong \sum_{i}\left(\frac{P_{i}}{P} \cdot \frac{1}{W_{i}}\right)
$$

where $P_{i}$ is the partial pressure of the $i$ th gas species, $P$ the total pressure of the mixture, and $P_{i} / P$ the partial pressure ratio of each type gas. The $W$ value has a slight dependency on the particle type but is considered as a constant value in our simulations. The $W$ value is 26.4 and $34.8 \mathrm{eV}$ for $\mathrm{Ar}$ and $N_{2}$, respectively [15]. The gas filled in BLMs used in CSNS is a mixture of 70\% argon and 30\% nitrogen, thus the $W$ value is about $28.7 \mathrm{eV}$ according to Eq. (3). Finally, the current signal of BLMs can be obtained by multiplying the result in terms of charge/primary with the beam loss intensity.

The calculations discussed above assume that the created charges in the sensitive volume are entirely collected, i.e., the collection efficiency is 1 . In general, the collection efficiency is indeed close to $100 \%$ supposing the bias voltage is in the ionization region, however, in practice collection efficiency is relevant to the dose rate which may bring about the space charge effect and the recombination loss [15]. The experimental investigations and brief discussions are presented below.

The saturation plateau of the ionization region ranges from 600 to $3500 \mathrm{~V}$ for the CSNS BLMs and the plateau slope is lower than $0.25 \% / 100 \mathrm{~V}$, which is tested by a radiation source ${ }^{60} \mathrm{Co}$ (details seen in Sec. II D). Figure 8 shows the test data of plateau curves, the data fit and the deduced collection efficiency of a BLM. The dose rates are respectively 360,48 , and $3 \mathrm{krad} / \mathrm{h}$. A semiempirical model proposed by Zankowski [28] is used to deduce the collection efficiency:

$$
1 / I=\left(1 / I_{\mathrm{sat}}+\alpha / V+\beta / V^{2}\right) e^{-\gamma V},
$$

where $V$ stands for the bias voltage, $I_{\text {sat }}$ the saturation current, $I$ the measured current, and $\alpha, \beta$ and $\gamma$ the fitting parameters. In theory, IC is operated in the ion chamber region where charge multiplication will not occur. However, in practice, multiplication is inevitable for bias voltage near the saturation region, although this effect is very slight. For a better description and fit of the experimental data, parameter $\gamma$ is introduced in Eq. (4) accounting for the charge multiplication effect. The fit formula follows well with the measured data as shown in Fig. 8(b). To calculate the collection efficiency, the charge multiplication should be excluded from the measured ionization 

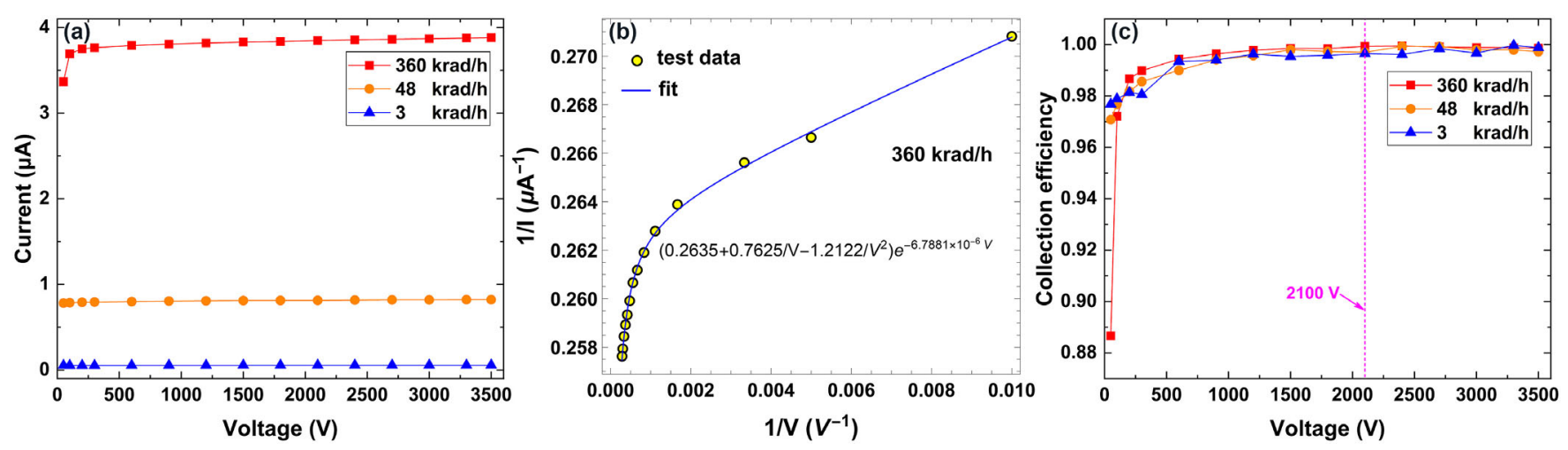

FIG. 8. (a) The test data of plateau curve, (b) the data fit, and (c) deduced collection efficiency of a BLM using a ${ }^{60}$ Co radioactive source. The dose rates are respectively 360,48 , and $3 \mathrm{krad} / \mathrm{h}$.

current. The deduced collection efficiency shown in Fig. 8(c) is higher than 0.99 for bias voltage greater than $900 \mathrm{~V}$, and there is no visible decline for dose rate rises from 3 to $360 \mathrm{krad} / \mathrm{h}$.

The response of IC with the radiation intensity will deviate from the linear relation as the ionization rate (ions created in unit time and volume) increases to a threshold value, which is induced by the recombination of the charge carriers. Boag proposed a recombination model for IC [29]; however, it is appropriate for the work gas containing an electronegative gas like air, not very suitable for a nonelectronegative gas such as argon or nitrogen. Zwaska proposed a recombination model for the nonelectronegative gas in his thesis [30], which is adopted to evaluate the collection efficiency of our BLMs. The space charge effect will deform the applied electric field, and a dead zone starts to form at a critical ionization rate given by

$$
\phi_{0}=\frac{4 \mu \varepsilon_{0}}{e} \cdot \frac{V^{2}}{d^{4}}\left[\frac{\text { ions }}{\mathrm{cm}^{3} \cdot \mu \mathrm{s}}\right],
$$

where $\mu$ is ion mobility, $\varepsilon_{0}$ the vacuum electric permittivity, $e$ the elementary charge, $V$ the bias voltage, and $d$ the electrode separation. For cylindrical geometry, the electrode separation uses the effective value

$$
d=\left[\left(a^{2}-b^{2}\right) \frac{\ln (a / b)}{2}\right]^{1 / 2},
$$

where $a$ and $b$ are the radius of outer and inner electrode, respectively [29]. The effective electrode separation is about $0.66 \mathrm{~cm}$ calculated from Eq. (6). The ion mobility $\mu$ for the binary gaseous mixture is given by Blanc's law [31]:

$$
\frac{1}{\mu_{\text {mix }}}=\frac{f_{1}}{\mu_{1}}+\frac{f_{2}}{\mu_{2}},
$$

where $\mu_{\text {mix }}$ is the mobility of mixture, $f_{1}$, and $f_{2}$ the volume fraction of gas 1 and 2, respectively. The mobilities $\mu_{1}$ and $\mu_{2}$ are the mobilities of the same ion in each of the gases. The mobility of argon and nitrogen ions is 1.7 and $2.67 \mathrm{~cm}^{2} /(\mathrm{V} \mathrm{s})$ [32], thus the ion mobility in the gas mixture of $\mathrm{Ar} / \mathrm{N} 2$ (70\%:30\% by volume) is about $1.91 \mathrm{~cm}^{2} /(\mathrm{V} \mathrm{s})$. Substituting all the parameters into Eq. (5), the critical ionization rate is $\sim 9.82 \times 10^{7}$ ions $/\left(\mathrm{cm}^{3} \mu \mathrm{s}\right)$, which corresponds to a charge of $1.73 \mathrm{mC}$ in one second in the sensitive volume of BLMs and is about 455 times higher than the maximum ionization rate in the above characteristics test experiments with ${ }^{60} \mathrm{Co}$ radioactive source, i.e., the space charge effects are negligible. Consequently, the collection efficiency degradation is almost invisible as shown in Fig. 8(c).

The ionization rate of BLMs in the radiation field of CSNS accelerator is however related to the beam loss patterns. The regular and irregular losses with different beam energies will give a wide range of ionization rate, and the ionization rate may exceed the critical value. The details of loss responses are presented in Sec. II F. For the case of the occurrence of space charge effects, the collection efficiency of IC based on the effective length can be derived as

$$
x_{0}=\left(\frac{4 \mu \varepsilon_{0}}{e} \cdot \frac{V^{2}}{\phi}\right)^{1 / 4}, \quad f=x_{0} / d,
$$

where $x_{0}$ is the effective length, $\varphi$ the ionization rate, and $f$ the collection efficiency. Equation (8) assumes all generated charges in the dead zone will be lost due to recombination, and the collection efficiency is considered as the ratio of the effective length to electrode separation.

In the practical design and application of BLMs, it is important to know which kind of secondary particles makes the major contributions to the response signal. We employ the two-steps method to study the contributions of different secondary particles to the total signal. The first step is to record the secondary particles position, direction, and energy on the monitor boundary (the boundary material is generally set as material "BLACKHOLE" in FLUKA in the first step), and all of these variables are considered as the phase space coordinates of secondary particles. The second step is to sample the secondary particles as 


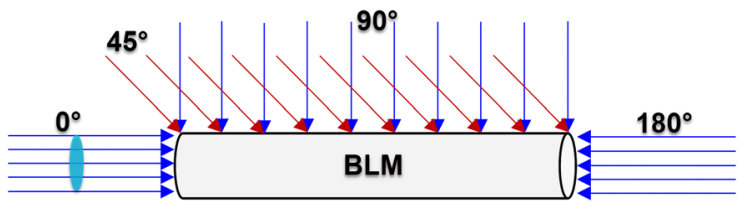

FIG. 9. The calculation model of response functions for particles traversing the BLM at four incident angles.

the incident particles from the particle phase space stored in the first step, then reimplement the particle's transportation process and record the BLM response. It should be noted that the boundary material must set as the real one in the second step. Both steps implemented in our FLUKA program need to write the user routines, routines as source.f and mgdraw.f are used and the corresponding SOURCE and USERDUMP card should be activated [21].

\section{The response functions of the CSNS BLM}

The ionization current offers the BLM output signal, which is determined by the particle intensity in the sensitive gas volume, the ionization cross section, and the effective range of particles. The ionization cross section and particle range both depend on the particle type and its energy, while the particle range is also relevant to the particle incident angle. The response functions can reflect all the dependencies, which provides the average charge produced in the sensitive volume by the particle traversing the BLM at different energies. The incident particles are uniformly distributed to traverse the detector, and then the BLM response is normalized to the number of incident particles and generally expressed in fC/primary. Due to the angle dependency, the different-angles investigation of response functions is a common method for the BLMs. This method was applied to the LHC BLM to obtain the response functions in previous researches, which was mainly performed with the Monte Carlo program GEANT4. The works were mainly given by Dehning, Stockner and Sapinski [22,33-35], and the simulation details are presented in Ref. [36].

As illustrated in Fig. 9, in order to obtain the response function, different particles traverse the BLM with different energies and angles. The particle beam is evenly distributed and has an identical cross section with the impact surface. We mainly research four representative angles: $0^{\circ}, 45^{\circ}$, $180^{\circ}$, and $90^{\circ}$. The degrees are with respect to the longitudinal axis of BLM, among which $180^{\circ}$ is incident on the end face having cable sockets, and $90^{\circ}$ is perpendicular to the BLM longitudinal direction. $270^{\circ}$ is omitted due to the BLM symmetry. The four angles are also the typical installation orientations with respect to the actual beam direction in the CSNS accelerator.

Figure 10 shows the response functions of the BLM. The kinetic energy of particles except neutrons ranges from $1 \mathrm{MeV}$ to $1.6 \mathrm{GeV}$, while the one for neutron ranges from
$10^{-11} \mathrm{MeV}$ to $1.6 \mathrm{GeV}$. Neutron response functions are separately shown in Fig. 10(e), and the enlarged view of $e^{ \pm}$ response functions is shown in Fig. 10(f). In general, responses of $0^{\circ}$ and $180^{\circ}$ have a higher value than the other angles because of the longer trajectory length for particles traversing the BLM. The curve tendency versus particle energy is similar to that of LHC BLM. However, the charge per primary expressed in $\mathrm{fC} /$ primary is slightly lower than LHC BLM in the same energy range. For example, the response of $40 \mathrm{MeV}$ photon impacting at $0^{\circ}$ for CSNS BLM is about $0.013 \mathrm{fC} /$ primary; this value is about $0.035 \mathrm{fC} /$ primary for LHC BLM, while this difference is not so large as energy increases [35]. The greater responses of LHC BLM could be attributed to its larger sensitive volume and much more electrodes that produce more secondary particles when the same energy primary particles traverse them.

Response functions can be divided into four groups based on the curve shapes: neutron; photon; $e^{ \pm}$; and proton, $\pi^{ \pm}, \mu^{ \pm}$. Response functions of proton, $\pi^{ \pm}$and $\mu^{ \pm}$follow a similar tendency that increases first then decreases. These heavy charged particles would have a threshold energy to penetrate the monitor wall. However, once their kinetic energy exceeds the threshold energy, the ionization cross section will decrease, that is to say, the peak formed in response functions is related to the Bragg peak [15]. The photon presents a smoother response and a simple increase tendency with the increase of energy. The electron and positron also give a smooth response as shown in Fig. 10(f). Although the electron and positron are charged particles, they will produce more secondary particles compared to other heavier charged particles that dissipate their energy mainly by ionization and excitation if all their incident energies are not enough to penetrate the monitor wall. Additionally, the electron and positron have almost the same responses except for the low energy section, which is caused by the effect of electron-positron annihilation [15]. The neutron is a neutral particle; it interacts with matter not directly by Coulomb force but the nuclear scattering or nuclear reaction, and the $(n, \gamma)$ capture reaction is the most common [15]. The general tendency of neutron responses decreases first and then increases with the increase of neutron energy. Neutrons with different energies undergo different reactions in different components of BLM, thus the response function is not so smooth compared with the photon. Take the small peak formed at $\sim 1.15 \mathrm{keV}$ for example, it is caused by the resonance neutron capture in the iron composition of the BLM stainless-steel shell or the electrodes $[37,38]$.

\section{D. $\gamma$-ray calibration experiment}

$\gamma$ rays of ${ }^{60} \mathrm{Co}$ source are utilized to calibrate BLMs and measure the ionization-induced current in this research. The purpose of the $\gamma$-rays calibration experiment is to measure the sensitivity of BLMs and validate the 

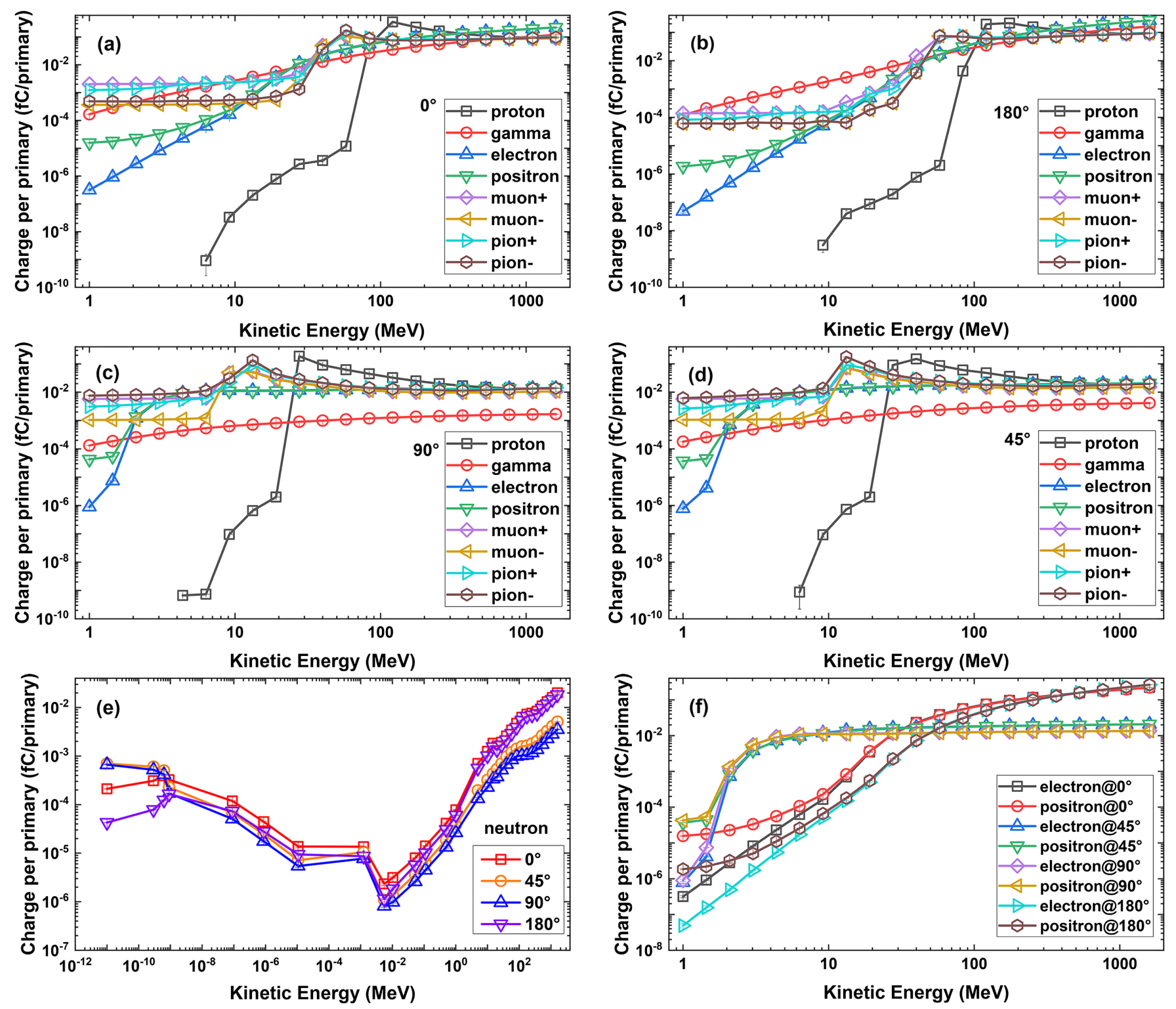

FIG. 10. Response functions for particles with different energies and angles. (a) $0^{\circ}$, (b) $180^{\circ}$, (c) $90^{\circ}$, (d) $45^{\circ}$, (e) neutron response functions in the range of $10^{-11} \mathrm{MeV}$ to $1.6 \mathrm{GeV}$, and (f) enlarged view of $e^{ \pm}$response functions.

correctness of Monte Carlo simulations. This experiment was carried out on the experimental platform in Beijing Normal University, and the radiation activity of ${ }^{60} \mathrm{Co}$ was about $2.92 \times 10^{14} \mathrm{~Bq}$ during the experiment. The ${ }^{60} \mathrm{Co}$ rod source was behind the BLM as shown in Fig. 11 during the irradiation experiment. The distance of BLMs with rod source can be changed to generate different dose rates, and the ionization current of BLMs was measured by a picoammeter. The rod source has a length of $43.2 \mathrm{~cm}$ and a diameter of $2.82 \mathrm{~cm}$, and the source capsule is made of $1 \mathrm{~mm}$ thick stainless steel. The multihole steel plate shown in Fig. 11 between the rod source and the BLM is to protect the source, and it has a thickness of $0.5 \mathrm{~mm}$. The linear attenuation coefficient of stainless steel for the ${ }^{60} \mathrm{Co}$ source is $0.426 \mathrm{~cm}^{-1}$ [39], thus the gamma attenuation in a $0.5 \mathrm{~mm}$ steel plate is $\sim 2 \%$; nonetheless, the actual attenuation is even lower due to the multihole structure. For simplicity, only the rod source, the source capsule, and the BLM are included in simulations, while the multihole steel plate and experimental hall are ignored. During the calibration experiments, the BLM was always parallel to the source as shown in Fig. 11. The distances between the BLM and the ${ }^{60} \mathrm{Co}$ source are $13,36,77,161,246$, and $328 \mathrm{~cm}$, respectively.

${ }^{60} \mathrm{Co}$ simultaneously decays to excited states of ${ }^{60} \mathrm{Ni}$ by $\beta^{-}$decay, then the deexcitation process of the ${ }^{60 *} \mathrm{Ni}$ nucleus emits two $\gamma$ rays with an energy of 1.33 and $1.17 \mathrm{MeV}$. The brief decay equation is as follows [15]: 


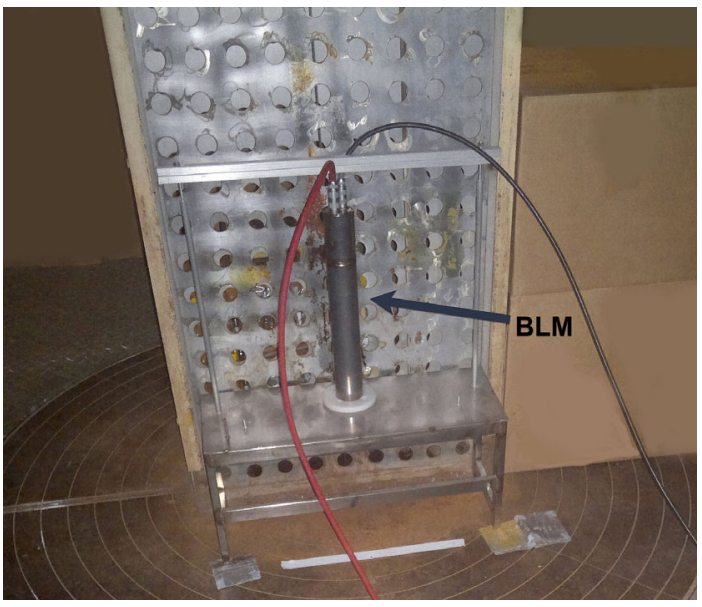

FIG. 11. The $\gamma$ ray irradiation experiment for the BLM using a ${ }^{60} \mathrm{Co}$ source. The source is hidden in a well when not used and lifted up during the experiment.

$$
\begin{aligned}
{ }_{27}^{60} \mathrm{Co} & \rightarrow{ }_{28}^{60 *} \mathrm{Ni}+e^{-}(0.318 \mathrm{MeV}) \\
& \rightarrow{ }_{28}^{60} \mathrm{Ni}+\nu_{e}+\gamma(1.332 \mathrm{MeV}, 1.173 \mathrm{MeV}),
\end{aligned}
$$

where $0.318 \mathrm{MeV}$ is the maximum energy of the continuous energy spectrum in $\beta$ decay. However, according to the response function shown in Fig. 10, the charge created in the BLM by $1-\mathrm{MeV} \gamma$ rays is about 2 orders of magnitude higher than that by electrons of the same energy, thus electrons of $0.318 \mathrm{MeV}$ will have a much lower contribution to the final signal compared with $\gamma$ rays of the two energies, so the electrons are neglected in the simulation. The two photons during one decay are assumed to emit isotropically in $4 \pi$ solid angle, and photon emitting positions are also uniformly sampled from the cylindrical volume.

Figure 12 shows the photon fluence distribution of the ${ }^{60} \mathrm{Co}$ source for the BLM at the distance of $36 \mathrm{~cm}$ (averaged over $5 \mathrm{~cm}$ ), which depicts the photon fluence of a rod source and shows a rapid descent of photon fluence with the increase of radial distance. The experimental and simulated ionization current are shown in Fig. 13, which presents a good conformity and indicates that the radiation-induced ionization current decreases dramatically with the increase of distance to the source. The results validate the correctness of the FLUKA program for the BLM response in a pure $\gamma$-ray radiation field. The maximum difference of measurement and simulation for a larger distance is about $15 \%$, and the statistical error of simulation is about $2 \%-3 \%$. The slight difference of simulation and experiments may be caused by the model simplifications, e.g., the experimental hall is not included in the simulation. The relatively larger difference for greater distance may also be relevant to the greater fluctuation at lower dose rates, e.g., the measurement uncertainty is $\sim 2.6 \%$ for distance below $77 \mathrm{~cm}$ while it reaches $\sim 6.4 \%$ at $328 \mathrm{~cm}$.

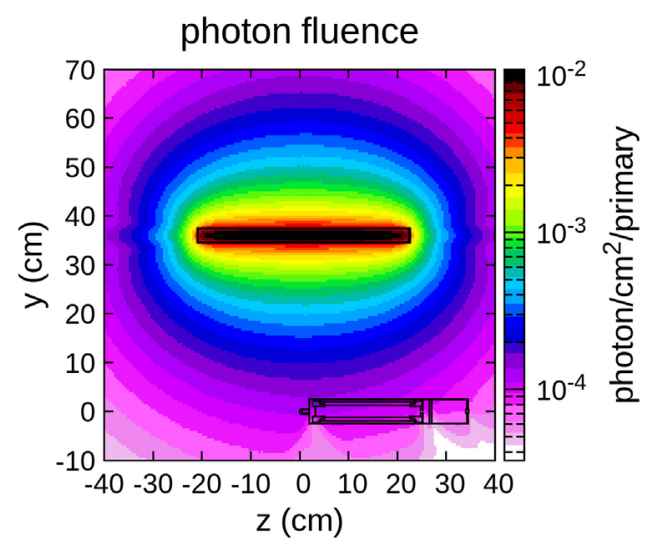

FIG. 12. The photon fluence map of ${ }^{60} \mathrm{Co}$ source. The distance of BLM to the source is $36 \mathrm{~cm}$, and the fluence map is averaged over $5 \mathrm{~cm}$.

\section{E. Beam loss measurement in the mixed radiation field and Monte Carlo validation}

Besides the $\gamma$ radiation experiment, simulations also need to be verified in a real mixed radiation field of a proton accelerating facility. A good way to validate simulations is to create an intentional loss and then measure the BLM response. Figure 14 depicts the temporary beam diagnostic system during the project construction. It includes a double-slit type emittance monitor and a Faraday cup (Fig. 14 only displays the first slit which samples the beam positions). They were placed at the exit of the DTL tank 1 where the $\mathrm{H}^{-}$beam energy was $21.67 \mathrm{MeV}$, and the current was $\sim 10 \mathrm{~mA}$. The temporary beam diagnostic system was removed before the remaining three DTL tanks were installed [40]. The emittance monitor consists of a 5 -mm thick high-grade polycrystalline graphite plate with a $0.1-\mathrm{mm}$ wide slit to sample the beam position.

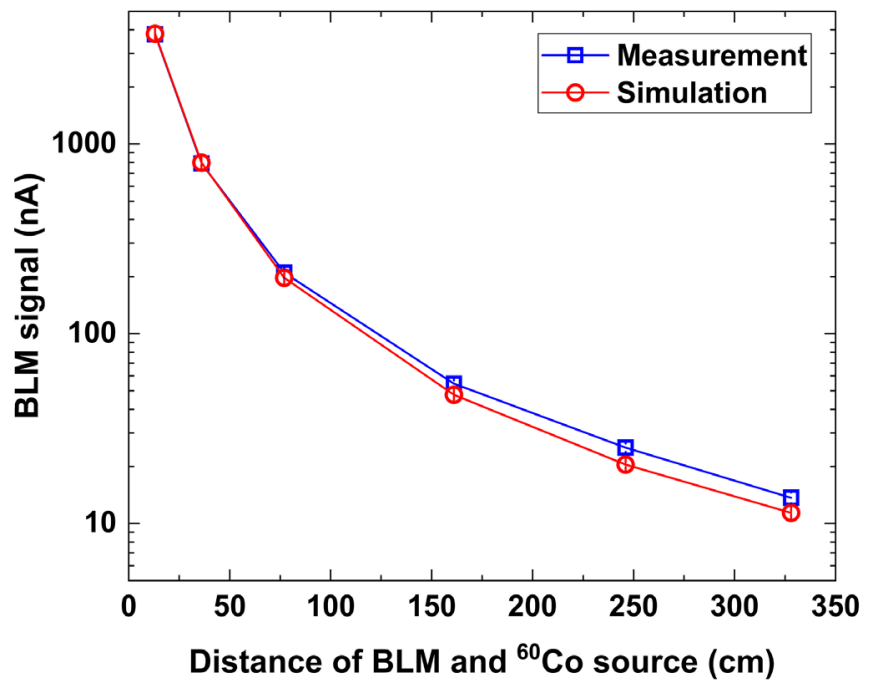

FIG. 13. The experimental and simulated BLM signal in the ${ }^{60} \mathrm{Co}$ radiation field at different distances. 


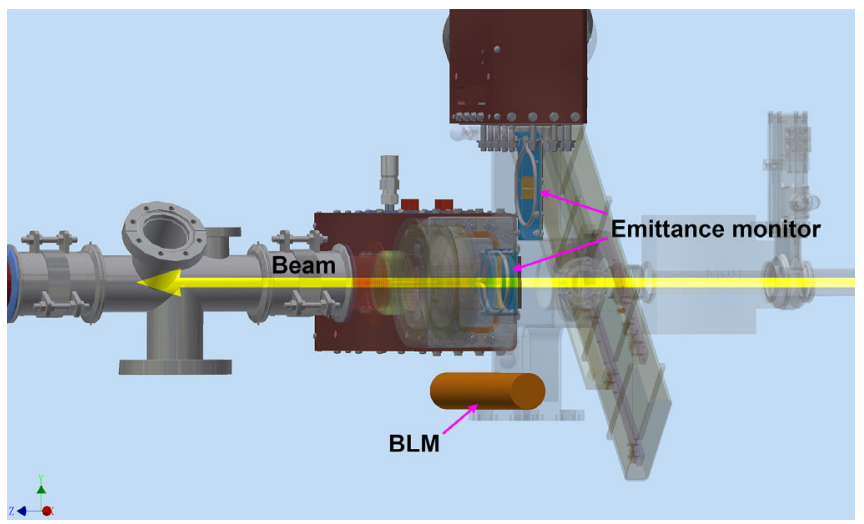

FIG. 14. The layout of the controlled beam loss experiment using an emittance monitor in the temporary beam diagnostic system of CSNS.

A $1.5 / 2.5 \mathrm{~mm}$ thick $\mathrm{Cu} / \mathrm{steel}$ plate was under the graphite plate and served as heat transferring and supporting structure respectively. Moving the emittance monitor could generate variable beam losses, and the lost beam current was obtained by the subtraction value of the currents measured by the forward and backward beam current transformers (CTs). During the experiment, the emittance monitor was moved in the $x$ direction to intercept the beam, the beam impact position was near the plate edge, and the moving range of the edge was about $2 \mathrm{~mm}$ relative to the pipe center. In simulations, the graphite plate is set as fixed due to the small variations in position. A pencil beam is assumed to impact near the edge; the simulation results of energy deposition in BLM are finally normalized to the ionization current according to the lost beam current. The accelerated particle is $\mathrm{H}^{-}$ion in our experiments. However, $\mathrm{H}^{-}$ions cannot be defined in FLUKA since only fully stripped protons and ions can be defined as beam particles for charged ions. Besides the reasons mentioned above, here we treat the $\mathrm{H}^{-}$ion as proton in FLUKA simulations because the two electrons energy of $\mathrm{H}^{-}$ion $(\sim 11.9 \mathrm{keV})$ is rather small compared to the proton energy. The two

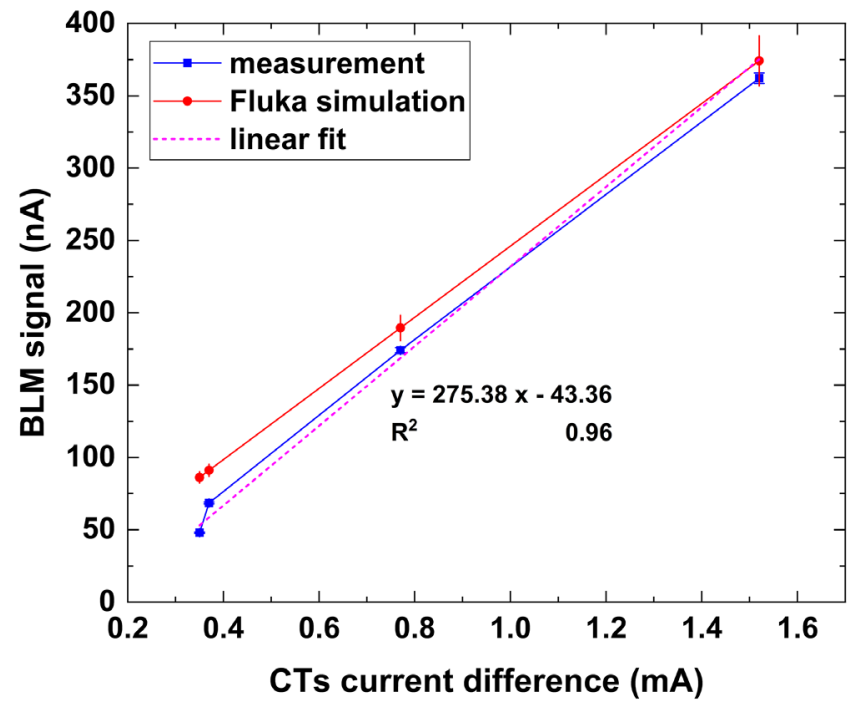

FIG. 16. Experimental and FLUKA simulated BLM signal in the mixed radiation field generated by the movable emittance monitor versus the beam current difference measured by CTs.

electrons can be easily stripped off by the graphite plate of the emittance monitor, during which turning the $\mathrm{H}^{-}$ion into proton. Compared with the electronic energy loss, the radiative losses start to dominate only for electrons typically up to tens of $\mathrm{MeV}$, more specifically, the critical energy is about $21.7 \mathrm{MeV}$ for iron, $19.4 \mathrm{MeV}$ for copper, and $81.7 \mathrm{MeV}$ for carbon respectively [41]. As a result, the 11.9-keV electrons mainly undergo the ionization energy loss process nearby the loss location, thus the contribution of stripped electrons to the BLM signal could be neglected.

Figure 15 displays the photon, neutron, and proton fluence nearby the vertical YZ plane (averaged over $1 \mathrm{~cm}$ ), which reveals the photon fluence is obviously higher and protons are entirely stopped in the vacuum chamber of the emittance monitor. Figure 16 shows the experimental and simulated BLM signal versus the lost beam current acquired by the CTs. Simulations are mostly
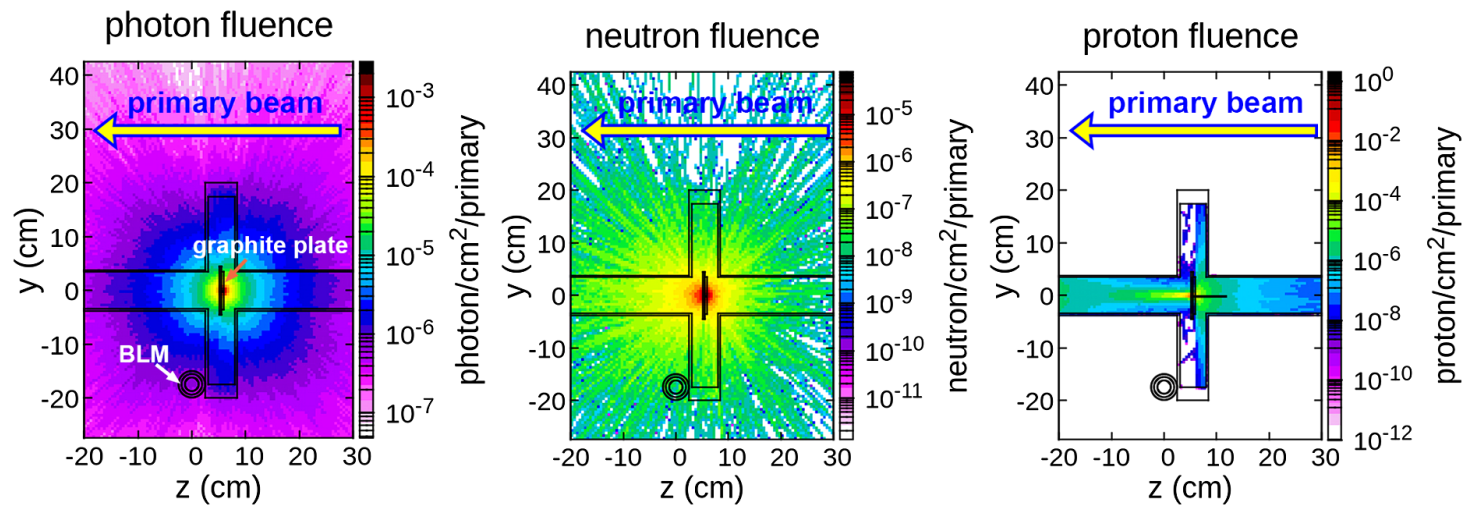

FIG. 15. The photon (left), neutron (middle), and proton (right) fluence distribution in the vicinity of the YZ plane (averaged over $1 \mathrm{~cm}$ ) for beam loss on the emittance monitor. 
TABLE III. Contributions from the different secondary particles to the signal for the controlled beam loss experiment with a movable emittance monitor.

\begin{tabular}{lc}
\hline \hline Particle type & Contribution to the signal \\
\hline Photon & $85.15 \%$ \\
Neutron & $0.057 \%$ \\
Electron & $14.04 \%$ \\
Positron & $0.753 \%$ \\
\hline \hline
\end{tabular}

consistent with the experimental results, both of which present an approximate linear relation with the lost beam current. Using the two-steps method the signal contribution of each particle can be obtained. The result is listed in Table III, it is obvious to see photons dominate the signal contribution, and following by electrons, positrons, and neutrons. There is no proton contribution since its energy is not sufficiently high to penetrate the vacuum chamber.

\section{F. FLUKA simulations for the beam loss responses and the different particle contributions to the signal}

BLMs detect the secondary particles produced by the interaction between the lost particles and the accelerator components. BLM responses to beam loss are related to the beam energy, beam loss patterns, mounting locations, surrounding objects, and even the BLM orientation. These dependencies are usually investigated through Monte Carlo simulations. However, some information is not always available, such as the detailed beam loss patterns, the loss locations, and the impact angle relative to the beam direction of the lost particles. As a result, some reasonable assumptions in the simulations have to be made for beam loss to simulate the BLM responses. Different loss scenarios are assumed to evaluate the BLM responses, which mainly involve the beam loss on drift tube in the linac part, loss on beam pipe of the straight section, quadrupole, and dipole in the RCS part. The uniformly distributed and localized losses are assumed to be the two main loss patterns in simulations. Losses are assumed to occur at a shallow angle relative to the beam direction except for an irregular loss at a dipole.

Figure 17 illustrates the loss scenarios, to conveniently discuss, the specific beam loss scenarios and contents of assumptions are discussed below.

Scenario I.- It simulates a grazing angle loss on the drift tube in the linac DTL part. The calculation model is modeled in detail with the drift tubes, the iron tank, and the BLM placed nearby the tank. The primary particle $\mathrm{H}^{-}$is replaced by the proton for the reasons presented in Sec. II E.

Scenario II.-It simulates a uniform loss at a grazing angle on the stainless-steel beam pipe of the straight section in the RCS part. The main geometric model is a 10-meters long beam pipe with an inner radius of $11.5 \mathrm{~cm}$ and a thickness of $0.2 \mathrm{~cm}$.

Scenario III.- It simulates a uniform loss at a grazing angle on the beam pipe across a quadrupole in the RCS part. The detailed model contains the magnetic poles, the iron yoke, and a beam pipe along the central line.

Scenario IV.-It depicts a grazing angle loss on the beam pipe at the longitudinal center of a quadrupole in the RCS

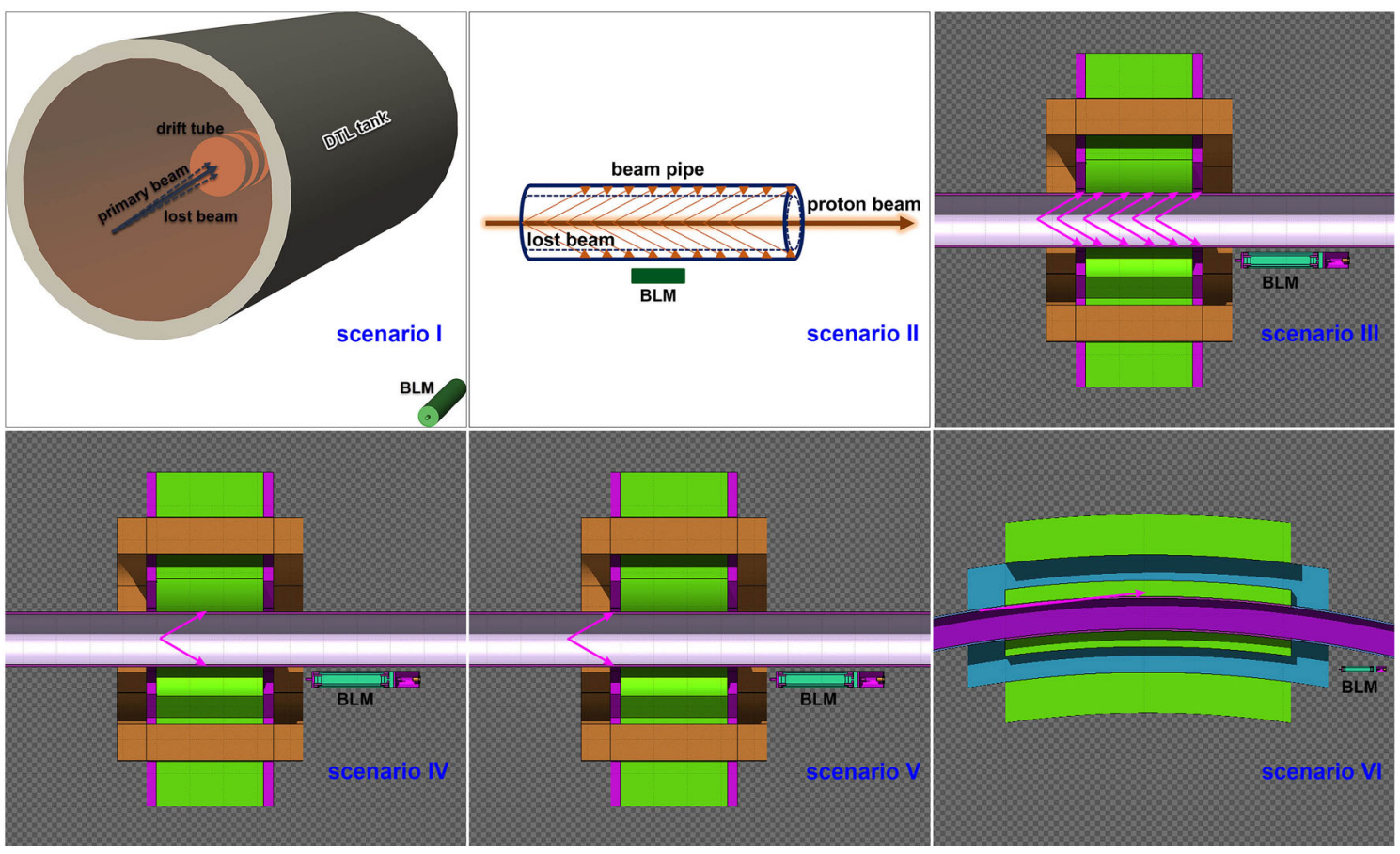

FIG. 17. Calculation models of the six assumed beam loss scenarios. 


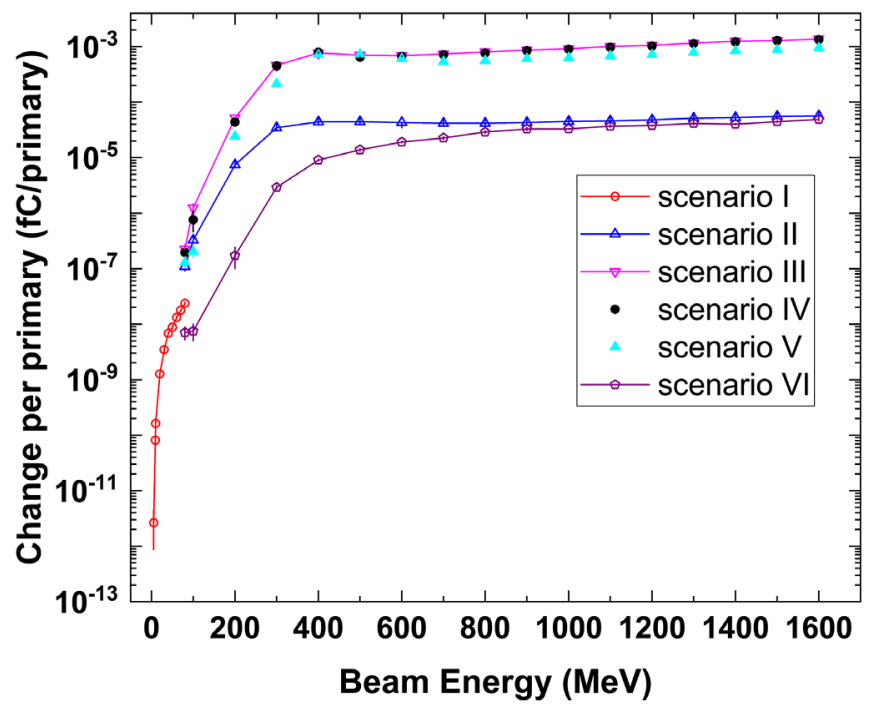

FIG. 18. BLM responses in the beam loss induced radiation field for the six loss scenarios.

part, which corresponds to the scenario of localized loss due to the higher beta-function.

Scenario V.-It depicts a grazing angle loss on the beam pipe at the entrance of a quadrupole magnet in the RCS part, which simulates the response differences due to a different loss location compared with scenario IV.

Scenario VI.-It depicts a localized loss on the beam pipe at the entrance of the main dipole magnet in the RCS part, which corresponds to an irregular beam loss caused by the beam misalignment or magnet malfunction. The direction of lost particles is parallel to the tangential direction of the beam orbit at the entrance of the dipole. A proton pencil beam is assumed to be lost, and strikes the pipe wall on the left.

Scenarios II and III simulate the BLM responses of uniform regular losses due to the residual gas scattering, space charge effect, or beam instability, etc. Scenarios I, IV, and $\mathrm{V}$ simulate responses of localized losses due to aperture limitations. Scenario VI simulates responses of an irregular loss caused by a malfunction of the bending magnet. For normal operational losses, the impact angle is expected to be rather small and considered to be lower than a few mrad in the CSNS accelerator. Without loss of generality and to simplify the calculations, the impact angle takes the value of $1 \mathrm{mrad}$ for the scenarios I-V (for comparison, the angle dependence for scenario III is also presented in the following). Beam energy ranges from 3-80 MeV in the linac part, and from $80-1600 \mathrm{MeV}$ in the RCS part. The quadrupole and dipole magnets for simulations are the frequently used types (RCS206Q, RCS160B) in the RCS part of CSNS.

For the uniform loss scenarios, we make the following detailed assumptions: (a) the lost beam strikes on the beam pipe at a rather small angle (1 mrad in our simulation); (b) the loss location is uniformly distributed along the beam direction, and the azimuth angle $(\varphi)$ of lost particles is also uniformly distributed in the range of 0 to $2 \pi$ [42]. Due to the small incident angle, it is inefficient to simulate if the lost particles emit from the pipe centerline, i.e., a lost particle will strike the beam pipe $r /(\tan \theta)$ downstream, where $r$ is the pipe radius and $\theta$ the impact angle. Therefore, to reduce the model size and improve the sampling efficiency, the lost particles are sampled from an imaginary cylindrical surface very close to the internal surface of the beam pipe [43]. The other loss scenarios are based on the similar assumptions (except scenario VI) except that the $z$ coordinate (in the beam direction) of loss point is fixed, which corresponds to a localized loss.

Figure 18 shows the BLM response per lost primary at different beam energy for the above six assumed scenarios. On the whole, the BLM response increases with increasing the beam energy for scenario I, while the response increases first then reaches a plateau for scenarios II-VI. Scenarios IV and V almost show the same response, and the slight difference is caused by the distance between the loss point and BLM. The BLM response ranges from the order of $10^{-12}$ to $10^{-8} \mathrm{fC} /$ primary for the beam energy from 5 to $80 \mathrm{MeV}$ (no response for $3 \mathrm{MeV}$ in the simulation) in the DTL part for scenario I, and from the order of $10^{-8}$ to $10^{-3} \mathrm{fC} /$ primary in the RCS part for scenarios II-VI. The BLM responses per primary are spread over a wide range of $10^{9}$ including all scenarios. This exceeds the dynamic range of the BLM readout system which is $10^{5}$.

Figure 19 exhibits the $2 \mathrm{~d}$ maps of energy deposition for beam loss scenario I at the beam energy of 10 and $80 \mathrm{MeV}$, and for scenarios II, III, and VI at the beam energy of $100 \mathrm{MeV}$ and $1.0 \mathrm{GeV}$ (from top to bottom). Maps are averaged over $4 \mathrm{~cm}$, and the white annular region (scenario I) or horizontal strip (scenarios II, III, VI) corresponds to the vacuum region where no energy is deposited. It is seen that the BLM signal is sensitive to the location from the contour map of energy deposition, and the energy deposited in the BLM is strongly dependent on the radial distance to the beam centerline. The energy deposition events are obviously rare for the beam loss at the energy of $10 \mathrm{MeV}$, where the shielding effect of the thick tank wall is very strong. So the energy deposition in BLM is much lower comparing to that of $80 \mathrm{MeV}$. This implies a common difficulty to detect the beam loss at the low energy section of a hadron accelerator. While along the RCS ring, where the beam energy is ramping from $80 \mathrm{MeV}$ to $1.6 \mathrm{GeV}$, a forward-directed characteristic is seen from the energy-deposition plots. This indicates that BLMs respond to not only the nearest beam loss, but also the secondary particles from further upstream.

Figure 20 indicates the impact angle dependence of BLM responses for scenario III, and the angle varies from 1 to $10 \mathrm{mrad}$. The variation tendency is similar for all angles, and the responses increase as the angle increases for all energies. Nonetheless, variations are not significant, the maximum difference for 1 and $10 \mathrm{mrad}$ is about a factor of 

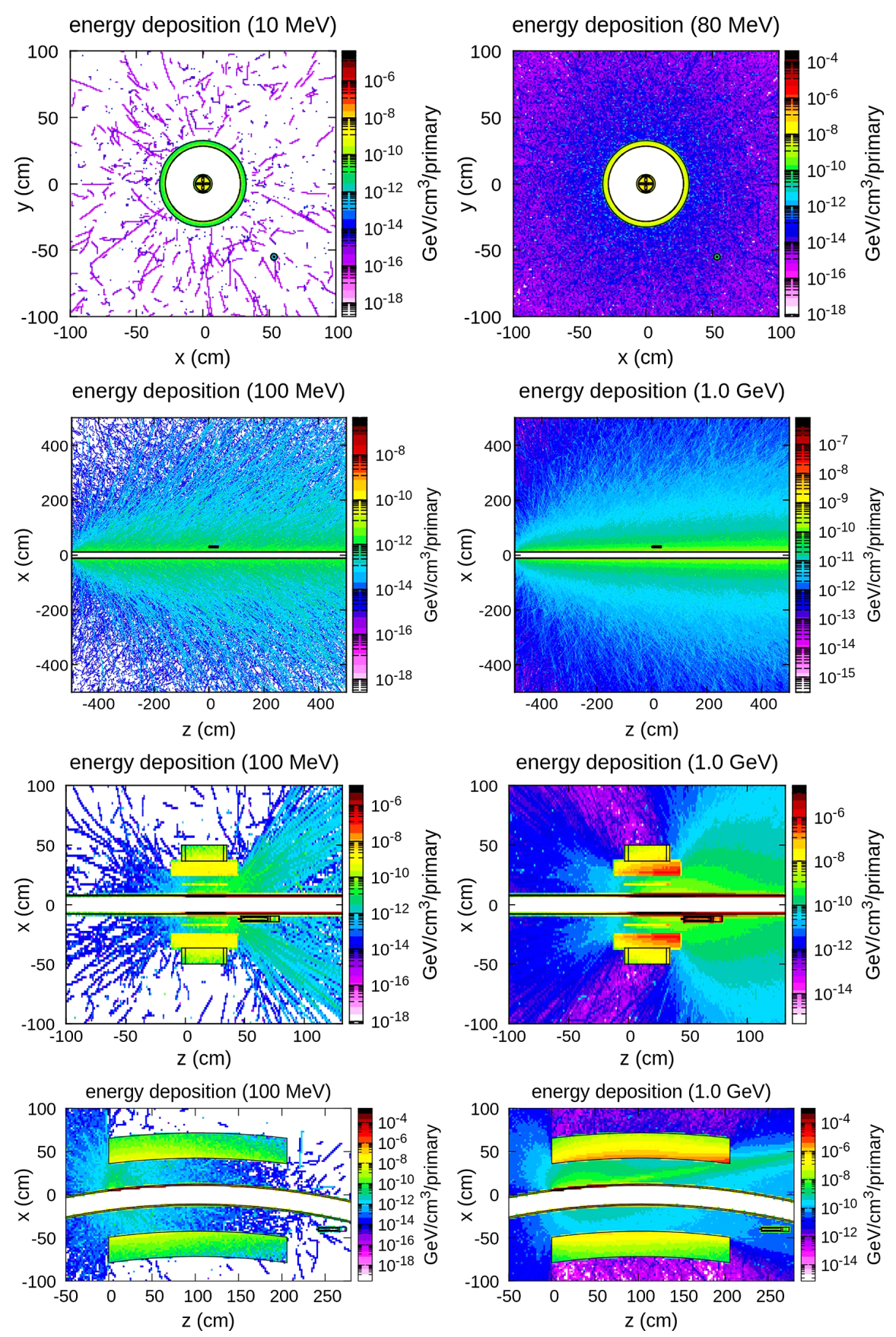

FIG. 19. Energy deposition maps in the XY plane for loss scenario I at the beam energy of 10 and $80 \mathrm{MeV}$, and maps in the XZ plane at the beam energy of $100 \mathrm{MeV}$ and $1.0 \mathrm{GeV}$ for the beam loss scenarios II, III and VI (from top to bottom row). Maps are averaged over $4 \mathrm{~cm}$, and the white annular region (scenario I) or horizontal strip (scenarios II, III, VI) corresponds to the vacuum region where no energy is deposited.

2 for beam energy greater than $300 \mathrm{MeV}$. Therefore, it is reasonable to select $1 \mathrm{mrad}$ as the impact angle to evaluate the BLM responses without resulting in an enormous discrepancy.

The beam loss signal is directly related to the composition and intensity of the secondary particles. Therefore besides the BLM overall responses to beam losses, in order to figure out the major contribution of the signal and the suitability of BLM type, it is also worth being concerned about the contributions of different particles to the signal. The so-called two-steps method is employed to study the issue, and the concrete procedure is introduced in Sec. II B. Figure 21 shows the energy deposition contributions of different particles for the six assumed scenarios. In the DTL part, the main contributions are from photons and neutrons due to the shielding effect of the tank to the secondary 


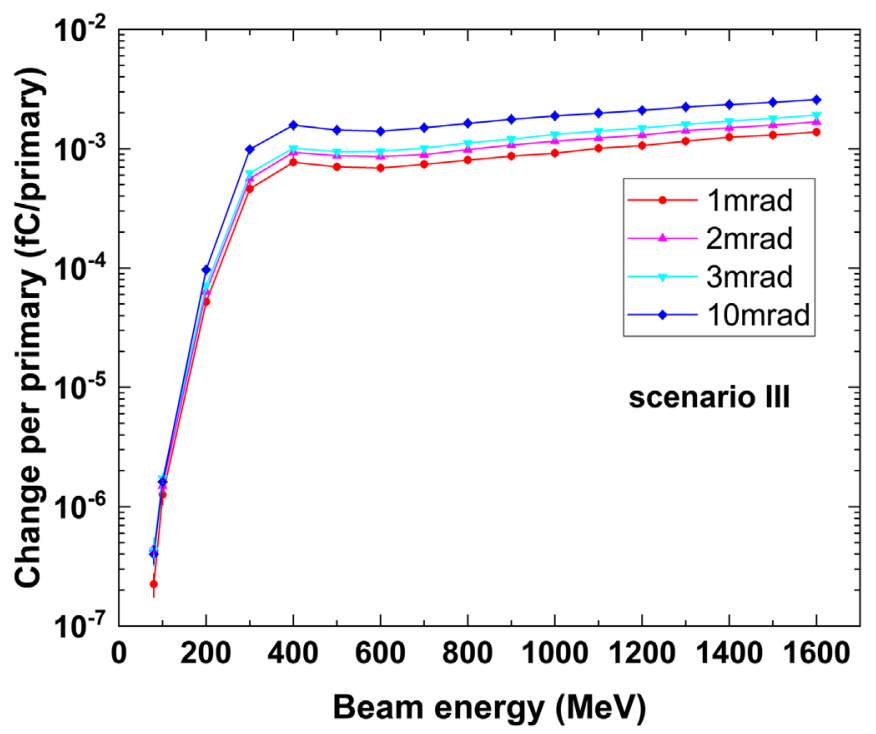

FIG. 20. The impact-angle dependence of BLM responses for scenario III, and the angle varies from 1 to $10 \mathrm{mrad}$.
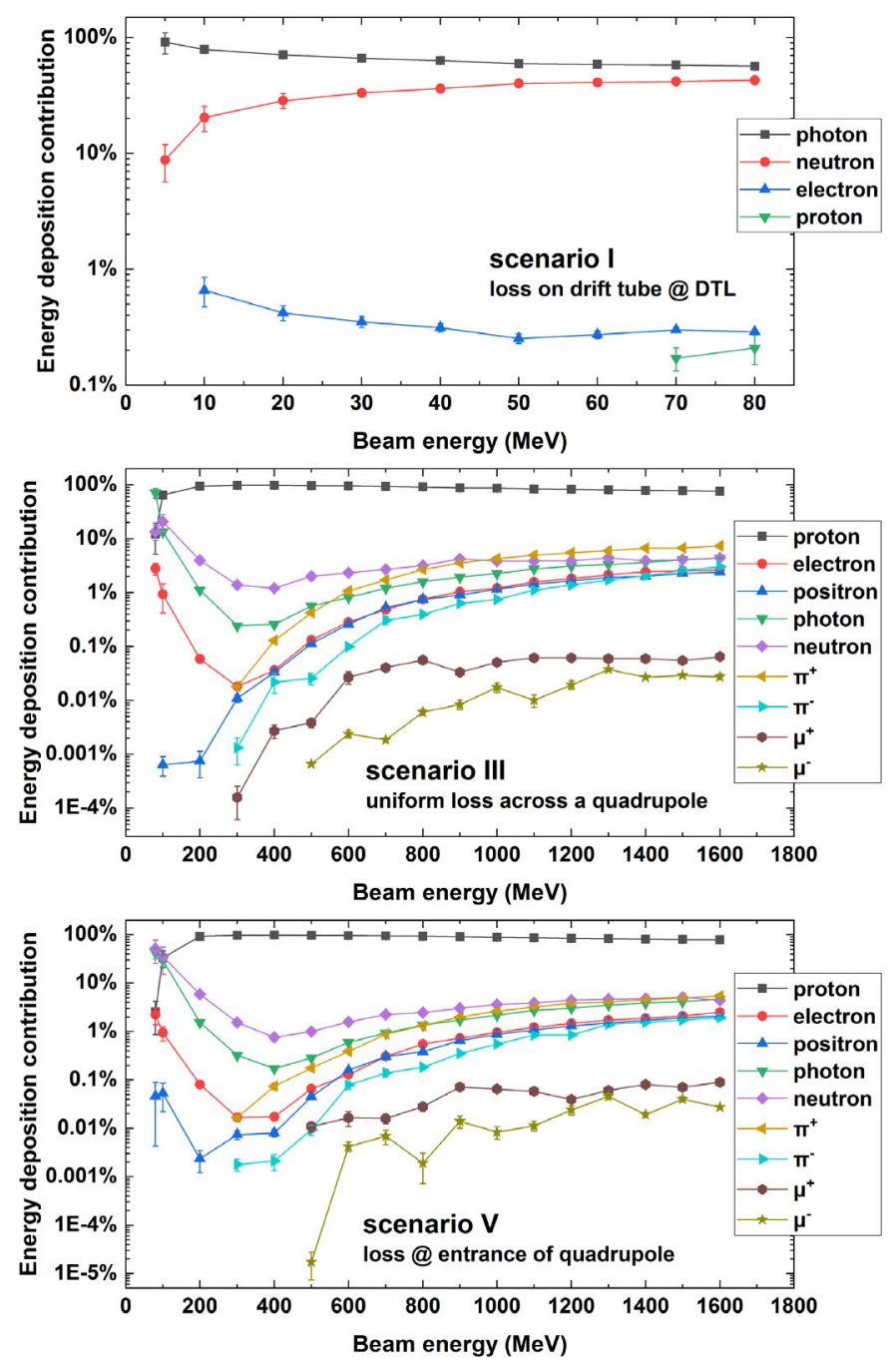

particles, especially for charged ones. The contribution of photons is greater compared with that of neutrons. Photons are generated from the $\gamma$ deexcitation process of the protoninduced inelastic nuclear reactions, or from the secondaryneutron induced capture reactions. In addition, the photon contribution decreases with the increase of beam energy. On the contrary, the neutron contribution increases for the reason that the energy and number of secondary neutrons increase for beam loss at higher energy. In the RCS part, the proton contribution dominates the total energy deposition for beam energy above $200 \mathrm{MeV}$. Scenarios II-VI show a similar tendency for proton contribution that increases first then decreases (falling from a peak of $\sim 90 \%$ to $\sim 70 \%$ at $1.6 \mathrm{GeV}$ ), and contributions from neutron, photon, and $e^{ \pm}$ roughly present an opposite tendency. The peak of proton contribution is at about $300-400 \mathrm{MeV}$. The contribution of protons goes down beyond that energy since the primary protons (which yield a higher contribution than secondary protons) are increasingly subject to nuclear interactions in the beam pipe because of the increased range since the
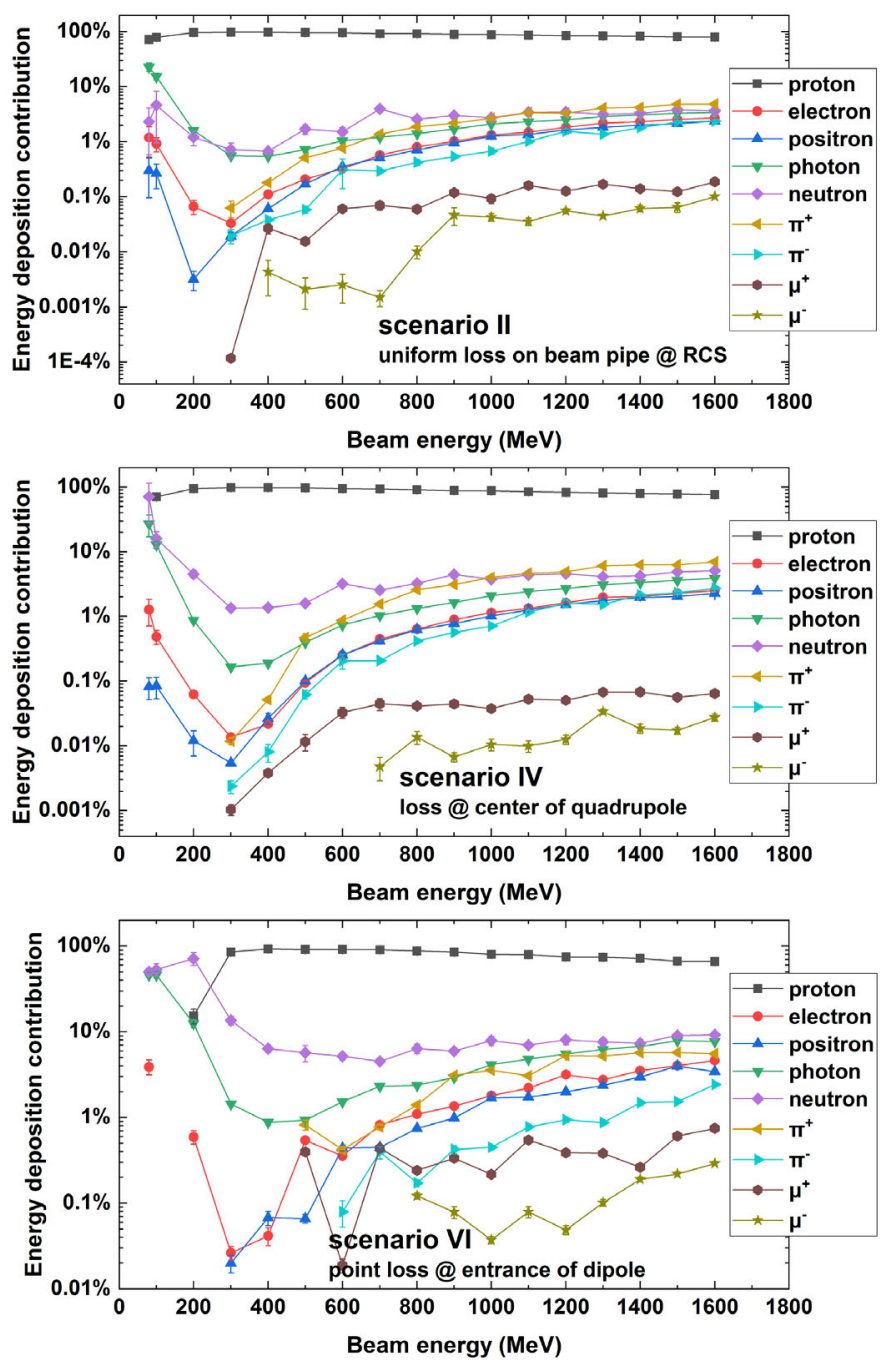

FIG. 21. Energy deposition contributions from different particles for the six assumed scenarios. 
cross section remains similar over a wide energy range. The buildup of the hadronic and electromagnetic shower will give rise to an increase of contributions from other secondary particles. The signal contribution of $\pi^{ \pm}$increases with the beam energy. Additionally, it has a positive value for beam energy higher than $\sim 300 \mathrm{MeV}$, which is determined by the production threshold of the $\left(p, \pi^{ \pm}\right)$reaction. The signal contribution from muons is rather small because of the low muon multiplicity (see Fig. 6), so it is reasonable to neglect the muon contribution in all of the beam loss scenarios.

In order to obtain the macroscopic ionization current responses of BLMs in different scenarios, the responses in terms of fC/primary should be multiplied with the beam loss intensity. $1 \mathrm{~W} / \mathrm{m}$ for a uniformly distributed beam loss is usually considered for most proton accelerators as an upper threshold. This criterion is based on the tolerable radiation exposure level for the hands-on maintenance of the proton accelerator [44]. Naturally, the beam-loss level of $1 \mathrm{~W} / \mathrm{m}$ is also an important design consideration not only for the radiation-shielding analysis, but also the beam loss monitoring. However, this criterion depends on the proton energy and is only a good approximation for energies above $100 \mathrm{MeV}$ for proton accelerators [44,45]. At lower energies, a greater number of particles can be lost as activation is less efficient [45]. Consequently, the beam loss rate is assumed to be $1 \mathrm{~W} / \mathrm{m}$ for scenarios II and III corresponding to a uniform loss in the RCS part. While in the DTL part, monitoring the loss of $1 \mathrm{~W} / \mathrm{m}$ is a harsh demand using the usual IC type of BLM due to the shielding effect of the DTL tank.

The lost beam induced heating damage is the most severe case in accelerator operations. So we focus on heating problems to evaluate the tolerable loss, which needs the finite element method (FEM) analysis. FLUKA calculations by using the USRBIN card can provide the inputs of the heat generation rate for the FEM approach [46], and the numerical FEM program ANSYS $®$ is adopted in this simulation. Heat conduction and thermal radiation are assumed as the cooling effects in the FEM calculations. The thermomechanical response of beam target interactions is a rather complex issue, and the problem is simplified by performing a Gaussian beam perpendicularly impacting on a block material. Copper is selected as the target material for its wide range of applications in the accelerator components such as magnet, drift tube, etc. Perpendicular incidence of a charged beam is apparently the worst case from the viewpoint of beam heating. Beam losses in RCS at the beam energy of $80 \mathrm{MeV}$ and $1.6 \mathrm{GeV}$ are selected as the scenario of beaminduced heating problems. The geometry implemented in simulations is a copper block with size $8 \times 8 \times 5 \mathrm{~cm}^{3}$ for $80 \mathrm{MeV}$ and $8 \times 8 \times 25 \mathrm{~cm}^{3}$ for $1.6 \mathrm{GeV}$, among which the 5 and $25 \mathrm{~cm}$ are the block thickness. The beam rms size is below a few millimeters in the CSNS RCS, which is assumed to be $1 \mathrm{~mm}$ in the thermal analysis for conservative considerations. The bunch time structure is introduced in Sec. I. For brevity, the problems of phase transitions are not involved in our discussions, our only concern is whether the temperature exceeds the melting point of the investigated material.

Figure 22 displays the energy deposition maps and the temperature distributions in copper for proton impacting at $80 \mathrm{MeV}$ and $1.6 \mathrm{GeV}$. In the case of $80 \mathrm{MeV}$, there exists a sharp peak at the longitudinal position $\sim 8 \mathrm{~mm}$ [nearby the projected range, see the left plot of Fig. 22(a)], which is known as the Bragg peak where the energy loss rate reaches the maximum. However, for the $1.6 \mathrm{GeV}$ case, no such sharp peak is formed and the maxima is close to the entrance [see the right plot of Fig. 22(a)]. The temperature for the case of $80 \mathrm{MeV}$ is approaching the melting point of copper $(1358 \mathrm{~K})$ just after 1.7 bunches $\left(1.33 \times 10^{13}\right.$ protons $)$ have been delivered in the first pulse [see the top left plot of Fig. 22(b)]. From the viewpoint of semiquantitative analysis, the specific energy from 298 to $1358 \mathrm{~K}$ is about $467 \mathrm{~J} / \mathrm{g}$ without regard to the latent heat of melting [47]. Figure 23 reveals specific energy loss for beam impacting the copper at $80 \mathrm{MeV}$ and $1.6 \mathrm{GeV}$ with beam rms size of $1 \mathrm{~mm}$, which indicates that 1.43 bunches at $80 \mathrm{MeV}$ are enough to elevate the temperature to the melting point from $298 \mathrm{~K}$. But considering the heat dissipation by thermal conduction in the adjacent zone around the Bragg-peak location, the required bunches are slightly more than the above rough estimation. For the case of $1.6 \mathrm{GeV}$, the peak temperature in a beam period reaches the maximum value of $624 \mathrm{~K}$ after 80 beam pulses [160 bunches delivered, see the bottom plot of Fig. 22(b)]. In other words, the beam-induced heating effect is more severe at a lower beam energy in the CSNS RCS. The final peak temperature is $477 \mathrm{~K}$ for $10 \%$ local loss at $80 \mathrm{MeV}$ after 100 pulses, which is also well below the melting point of copper [see the top right of Fig. 22(b)]. Similar FEM analysis is also performed at lower energy than $80 \mathrm{MeV}$ (linac scenario) for $10 \%$ local loss, which also indicates the temperature elevation is within the safe range.

Based on the above analysis, it can be concluded that a $0.1 \%$ loss of beam bunch will not bring about heat damage for the accelerator components in the CSNS. This loss rate is assumed for scenarios I, IV, and V, however, it is noted that this loss rate is only taken as the fairly representative value, and the BLM responses can easily be scaled to different loss rates. The full loss of beam bunch for scenario VI is a hypothetical extreme case, which represents the worst possible accident caused by the failure of the bending-magnet power supply system. In simulations of BLM current, the peak current in beam duration is assumed to be $15 \mathrm{~mA}$ in the linac. In RCS the proton amount in one bunch is $0.78 \times 10^{13}$ and the beam duration is fixed to 500 ns neglecting the bunch compression for simplification. The ionization rate is calculated with the energy deposition in the BLM sensitive volume divided by the 

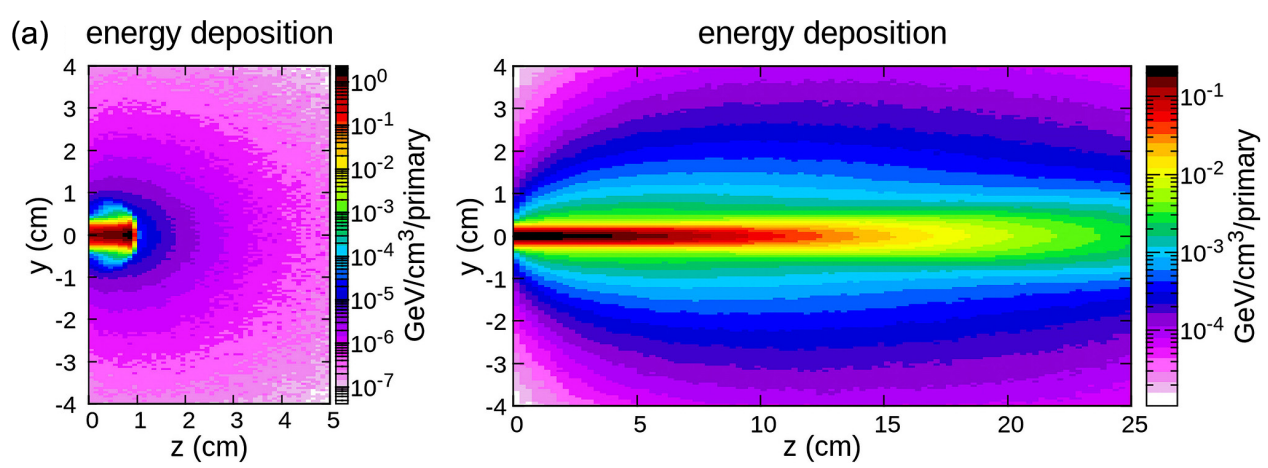

(b)

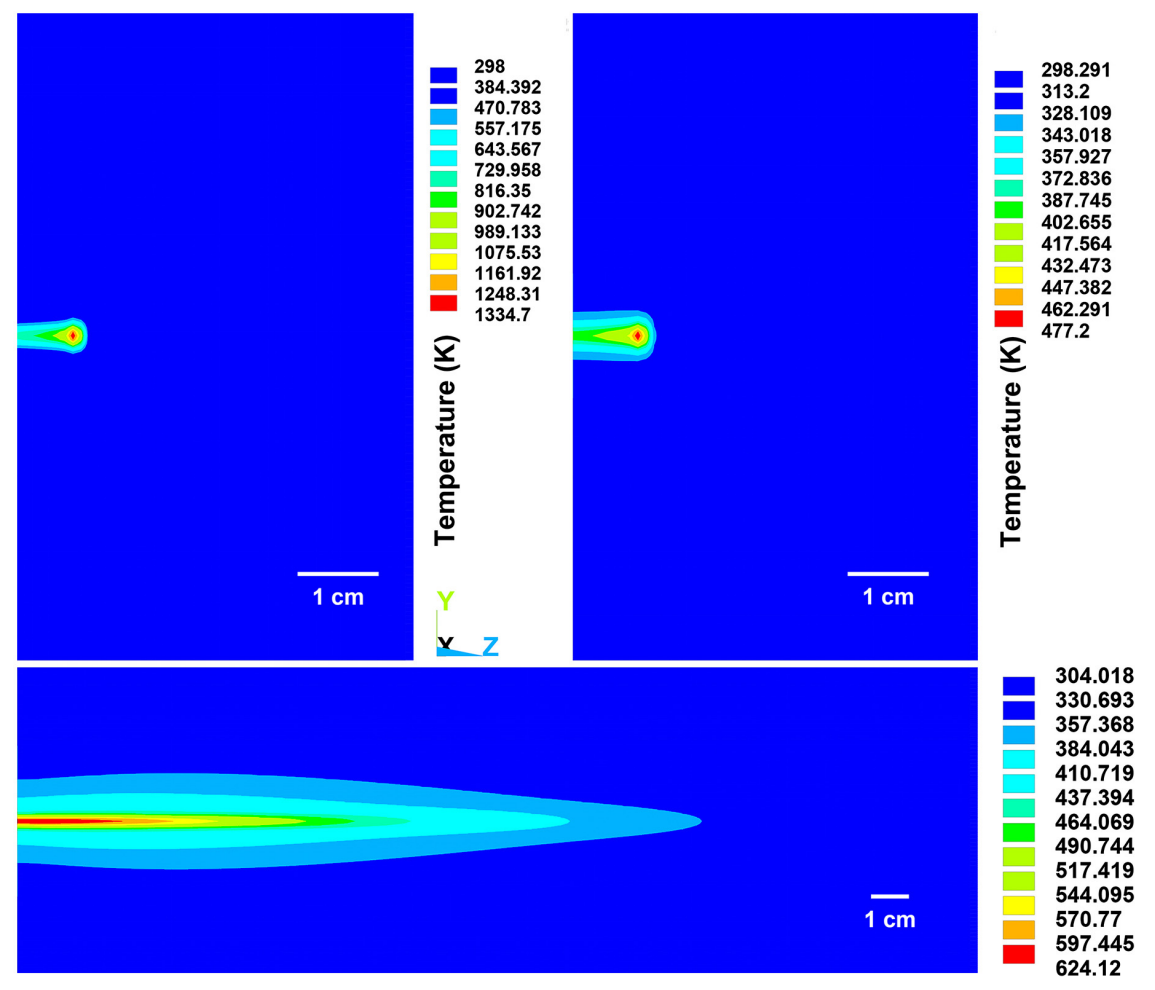

FIG. 22. (a) Energy deposition maps for proton impacting at $80 \mathrm{MeV}$ (left) and $1.6 \mathrm{GeV}$ (right). (b) The temperature distribution in copper for proton impacting at $80 \mathrm{MeV}$ just after 1.7 bunches delivered (top left) and 100 bunches delivered at $10 \%$ loss rate (top right), and the bottom plot is the case of $1.6 \mathrm{GeV}$ just after 80 bunches delivered.

volume and beam duration, and then the collection efficiency is obtained by Eq. (8).

The simulated current results for scenarios IV and V with the $0.1 \%$ loss and scenario VI with full loss are shown in Fig. 24, and the dashed lines correspond to the corrected current due to the space charge effects. The current for $0.1 \%$ loss of scenario I increases with the beam energy and ranges from 0.25 to $2222.09 \mathrm{pA}$. Although simulations get a positive result for beam energy below $20 \mathrm{MeV}$, however, due to the sensitivity of electronics, this beam loss signal could not be efficiently detected if the loss rate is very low. For example, the simulated BLM current is only about $0.25 \mathrm{pA}$ at the loss rate of $0.1 \%$ when the beam energy is $5 \mathrm{MeV}$, which is too small to be detected and it is also confirmed during the beam commissioning and machine operation. In practice, beam loss can be hardly detected for beam energy below $20 \mathrm{MeV}$ during the actual machine operation in CSNS, which is consistent with the simulated results. Considering of $200 \mathrm{pA} \sim 20 \mu \mathrm{A}$ the dynamic range of the BLM electronics used in CSNS, BLMs could detect a $0.1 \%$ loss level except for the low energy section $(<20 \mathrm{MeV})$ in DTL. The simulated current for $1 \mathrm{~W} / \mathrm{m}$ loss of scenarios II and III increases first and then declines with the increase of beam energy, and the current range falls within the electronics dynamic range and in the order of $\mathrm{nA}$ except for the beam energy below $200 \mathrm{MeV}$. Because the BLM response per primary shows a plateau region for beam energy greater than $300 \mathrm{MeV}$ (shown in Fig. 18), the loss rate of $1 \mathrm{~W} / \mathrm{m}$ means fewer protons for higher beam energy, which corresponds to the slight decline tendency for the $1 \mathrm{~W} / \mathrm{m}$ loss of scenarios II and III. In addition, the ionization rates of scenarios I-III with the respective loss 


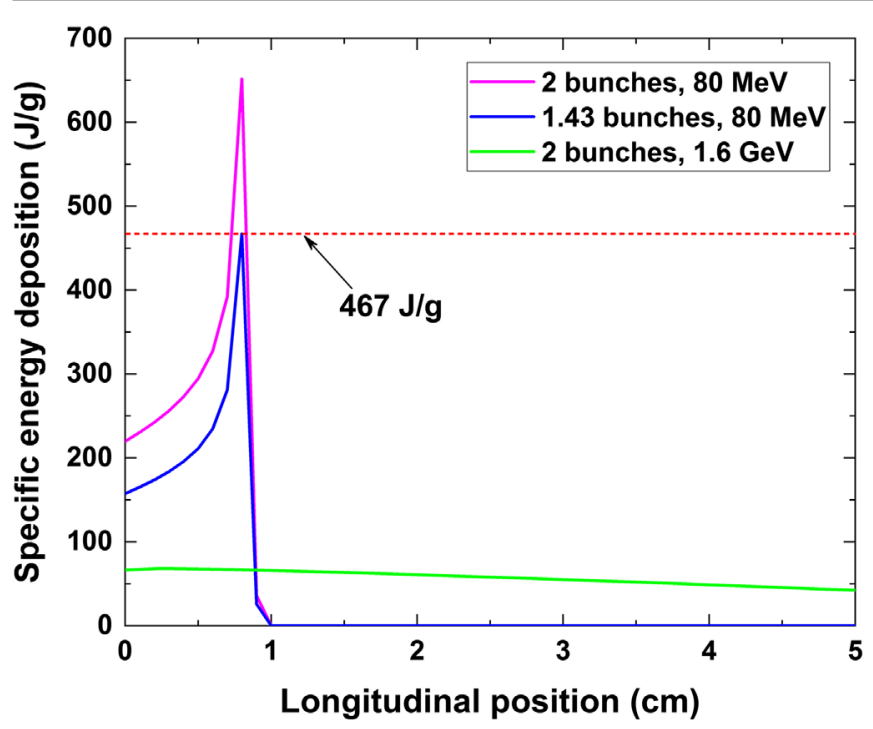

FIG. 23. Specific energy loss for beam impacting the copper at $80 \mathrm{MeV}$ and $1.6 \mathrm{GeV}$ with beam rms size of $1 \mathrm{~mm}$.

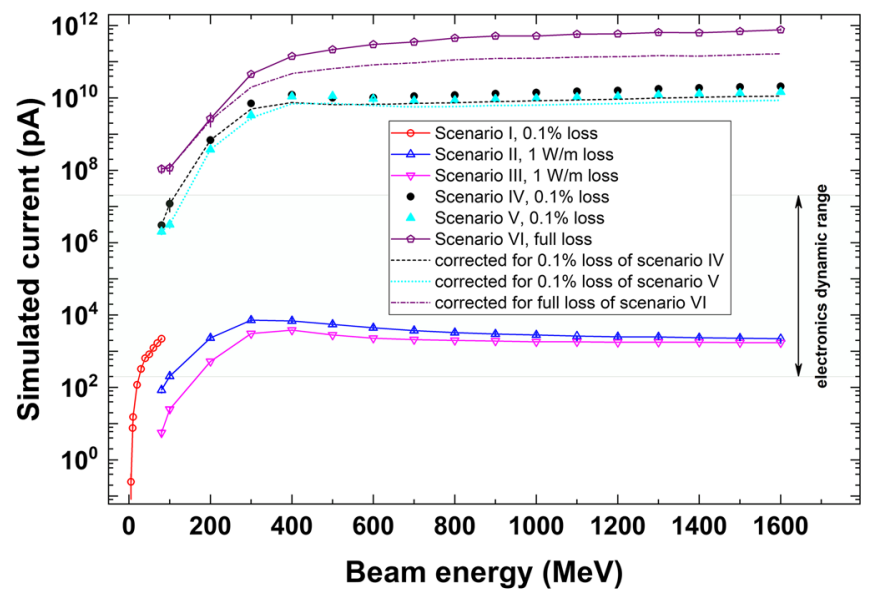

FIG. 24. Simulated ionization current for scenarios IV and V with a $0.1 \%$ loss and scenario VI with full loss, and the dashed lines correspond to the corrected current due to the space charge effects.

rates are far below the critical value presented in Sec. II B, so the current correction due to the space charge effect is needless.

Current responses for $0.1 \%$ loss of scenarios IV and V and full loss of scenario VI increase first and then reach a plateau, which is consistent with the response function per lost primary due to the fixed fraction of beam loss. The simulated current for scenarios IV and V with $0.1 \%$ loss is far beyond the upper limit of input of electronics except for the beam energy below $200 \mathrm{MeV}$, which however is not a serious issue from a monitoring perspective since this overthreshold signal will trigger the MPS to shut down the beam. Disregarding the saturation of electronics, the ionization rate already exceeds the critical value for the three scenarios with the corresponding loss rates when the beam energy is greater than $200-300 \mathrm{MeV}$, and meanwhile space charge effects begin to play an important role. The dashed lines depict the corrected currents, the collection efficiency declines from 0.70 to 0.53 for scenario IV with $0.1 \%$ loss when the beam energy rises from $300 \mathrm{MeV}$ to $1.6 \mathrm{GeV}$, and it declines from 0.85 to 0.59 for scenario $\mathrm{V}$ with $0.1 \%$ loss within the same energy range, while for the case of full loss of scenario VI, the collection efficiency declines from 0.89 at $200 \mathrm{MeV}$ to 0.22 at $1.6 \mathrm{GeV}$. The loss rate on the order of $\sim 10^{-5}$ in one bunch for scenarios IVVI will give a current range within the electronics dynamic range as shown in Fig. 24, nevertheless, this loss rate would not bring about the space charge effects. In other words, the input of the BLM electronics will be saturated before the space charge effects could occur. Furthermore, the use of additional BLMs with less sensitivity or more distance relative to the beam line helps in avoiding saturated signals and increases the dynamic range of the BLM system.

It should be realized that the BLM signal is sensitive to the beam loss details, the installation locations, and the surrounding objects. Nevertheless, the accurate loss patterns and loss locations in practical situations are not always known in advance, so the accurate prediction of the BLM signal is rather difficult. We mainly give the approximate signal range based on some assumed loss scenarios in this section. In the simulations for a specific beam loss experiment, more details of beam loss should be clear as the controlled beam loss experiment presented in Sec. II E.

\section{BEAM LOSS DETECTION IN THE LOW ENERGY SECTION OF THE CSNS LINAC}

Beam loss detection in the low energy section of the CSNS linac (i.e., $<20 \mathrm{MeV}$ ) is a difficult task using our $\mathrm{Ar} / \mathrm{N}_{2}$ IC since the thick DTL tank $(\sim 4.2 \mathrm{~cm})$ has a significant shielding effect on the beam-loss induced secondary particles. The secondary particles cannot have a considerable possibility to penetrate the tank wall. However, more or fewer neutrons will leak out of the tank, which may be easier to be detected if the neutrons are converted to charged particles. Based on different detection demands and neutron converters, different types of neutronsensitive detectors are used as the BLMs in several accelerator laboratories [48-50]. In the preliminary stage, we decide to detect the thermal neutrons as an evaluation of the beam loss level. As is known to all, ${ }^{3} \mathrm{He}$ and ${ }^{10} \mathrm{~B}$ are the most frequently used isotopes to detect thermal neutrons. For thermal neutrons with the kinetic energy of $0.025 \mathrm{eV}$, the reaction cross section is respectively 5333 barns for ${ }^{3} \mathrm{He}$ and 3837 barns for ${ }^{10} \mathrm{~B}$ [51]. Due to the present high price of ${ }^{3} \mathrm{He}$ gas [52], $\mathrm{BF}_{3}$ gas is employed as the neutron converter and $\mathrm{PE}$ as the neutron moderator, and the boron we use is $96 \%$-enriched ${ }^{10} \mathrm{~B}$. The relevant nuclear reaction is as follows [15]: 


$$
n+{ }^{10} \mathrm{~B} \rightarrow \begin{cases}\alpha+{ }^{7} \mathrm{Li}+2.31 \mathrm{MeV}+\gamma(0.48 \mathrm{MeV}) & 94 \% \\ \alpha+{ }^{7} \mathrm{Li}+2.79 \mathrm{MeV} & 6 \% .\end{cases}
$$

The dominant reaction releases total kinetic energy of $2.31 \mathrm{MeV} ; \alpha$ or $\mathrm{Li}^{3+}$ ions may deposit all their kinetic energy depending on their initial direction in the sensitive volume of the BLM. Ions have a large specific energy loss in the work gas compared to neutral particles, so a considerable signal from the $\mathrm{BF}_{3}$ monitor could be expected if we reasonably design and assemble the monitor.

Figure 25 depicts the experimental layout and the FLUKA geometric model of the two types of BLMs installed near the DTL. BLMs are installed at the $2 / 3$ of the first tank length where the nominal beam energy is about $15 \mathrm{MeV}$. The $\mathrm{BF}_{3}$ monitor is enclosed by a $7.5-\mathrm{cm}$ thick $\mathrm{PE}$ moderator, for comparison, an $\mathrm{Ar} / \mathrm{N}_{2} \mathrm{BLM}$ is placed close to the $\mathrm{BF}_{3}$ one. The monitors' responses are acquired to validate the feasibility of the scheme.
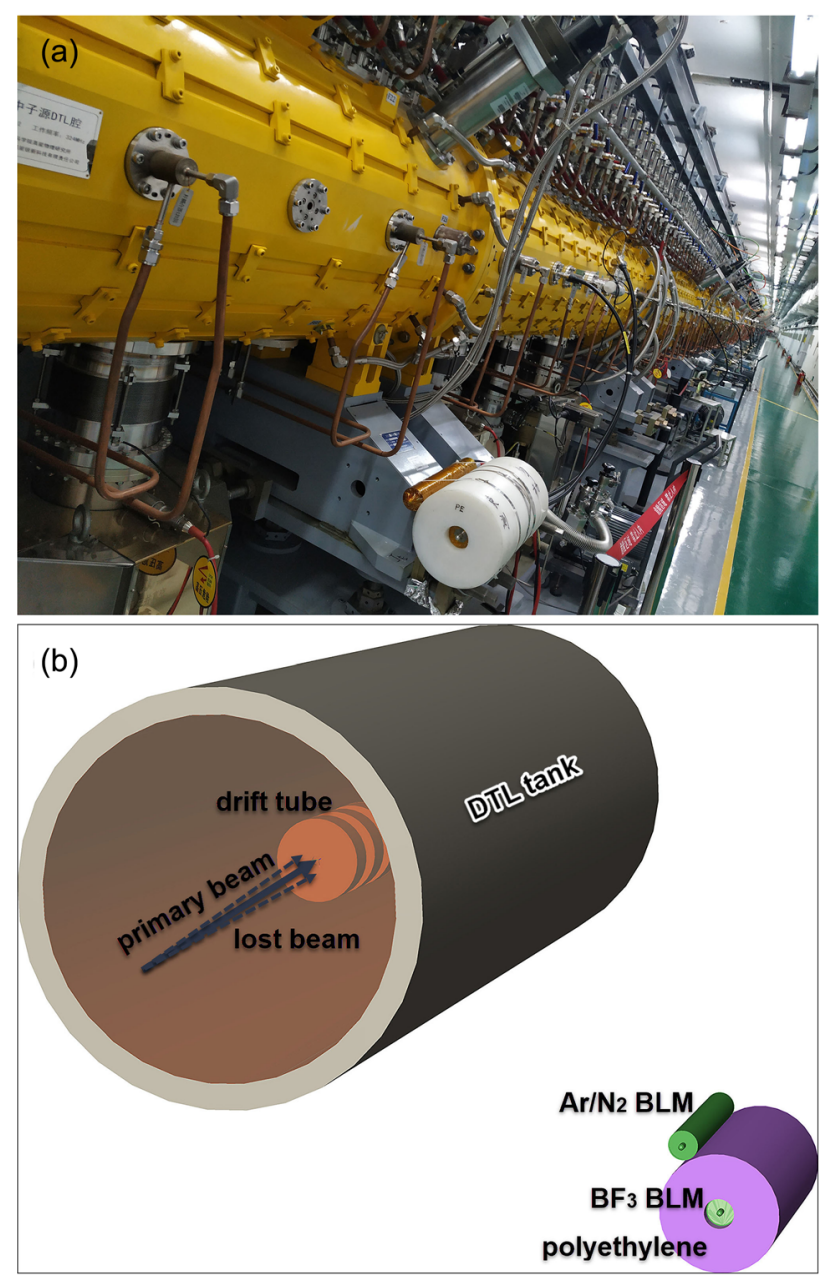

FIG. 25. (a) Experimental layout of two types of BLM installed at the first tank of DTL, and (b) the geometric model implemented in FLUKA.
Figure 26 reveals the simulated spectra of neutrons entering into the two BLMs, which presents an apparently higher thermal-neutron peak for the $\mathrm{BF}_{3}$ BLM. The spectrum softening of $\mathrm{BF}_{3} \mathrm{BLM}$ is caused by the neutron thermalization process in the PE. It is worth noting that the neutron spectrum of $\mathrm{Ar} / \mathrm{N}_{2} \mathrm{BLM}$ is modulated by the backscattered neutrons from the PE moderator of $\mathrm{BF}_{3}$ BLM, which is confirmed by removing the PE moderator. The consequent neutron spectrum shows no peak of thermal neutrons, and only fast neutrons enter into the $\mathrm{Ar} / \mathrm{N}_{2} \mathrm{BLM}$. The kinetic energy of PE-moderated neutrons ranges from $10^{-3}$ to $10^{7} \mathrm{eV}$, thus the cross section of the ${ }^{10} \mathrm{~B}(n, \alpha)^{7} \mathrm{Li}$ reaction spans from about 1.5 to $2 \times 10^{4}$ barns [53]. The simulated energy deposition in the sensitive volume of two BLMs is respectively $0.1335( \pm 2.1 \%) \mathrm{eV} /$ primary and $1.115 \times 10^{-4}( \pm 7.1 \%) \mathrm{eV} /$ primary for the $\mathrm{BF}_{3}$ and $\mathrm{Ar} / \mathrm{N}_{2}$ monitor. Thus the beam loss signal of the $\mathrm{BF}_{3}$ monitor is increased by a factor of $1197( \pm 89)$, which is a considerable amplification for the signal. The relevant experiment was carried out during the beam commissioning. Figure 27 shows the waveforms of the two monitors in a same beam loss event, which shows an obvious larger beam loss signal for the $\mathrm{BF}_{3}$ monitor. However, due to the possible very small loss ratio, the $\mathrm{Ar} / \mathrm{N}_{2}$ monitor cannot detect an effective signal. The $\mathrm{BF}_{3}$ monitor signal reaches a saturation level [see Fig. 27(b)] because of the saturation voltage of the operational amplifier. Due to the integral circuit for BLM electronics and the bandwidth in combination with the electronics, the signal waveform of beam loss has a long rising edge. In the beam pulse duration, the BLM signal will keep going up and not reach the flattop. To quantitatively investigate the signal magnification factor of the $\mathrm{BF}_{3} \mathrm{BLM}$ relative to $\mathrm{Ar} / \mathrm{N}_{2}$ one, the waveform fitting is essential. An unsaturated waveform is selected to fit, according to the pulse shaping theory, the unsaturated data is fitted by applying the equation as follows [15]:

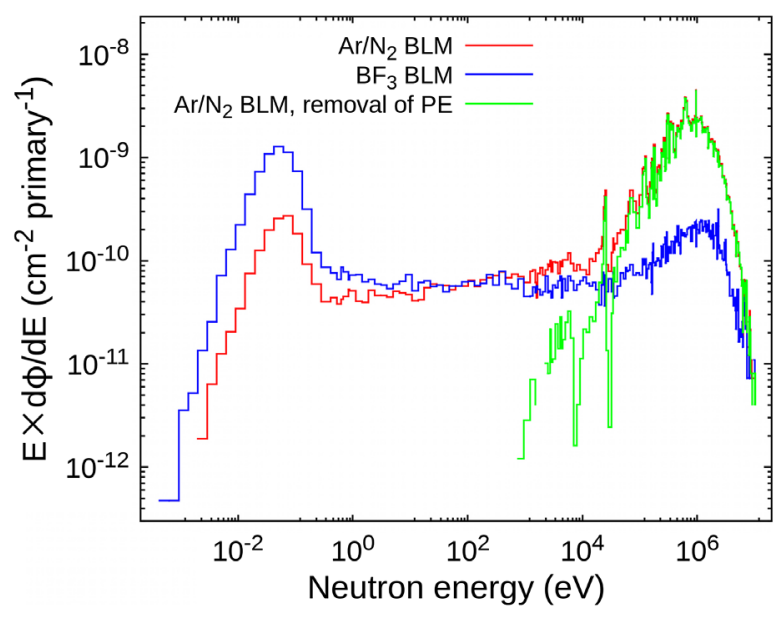

FIG. 26. Simulated spectra of neutrons entering into the two BLMs. 

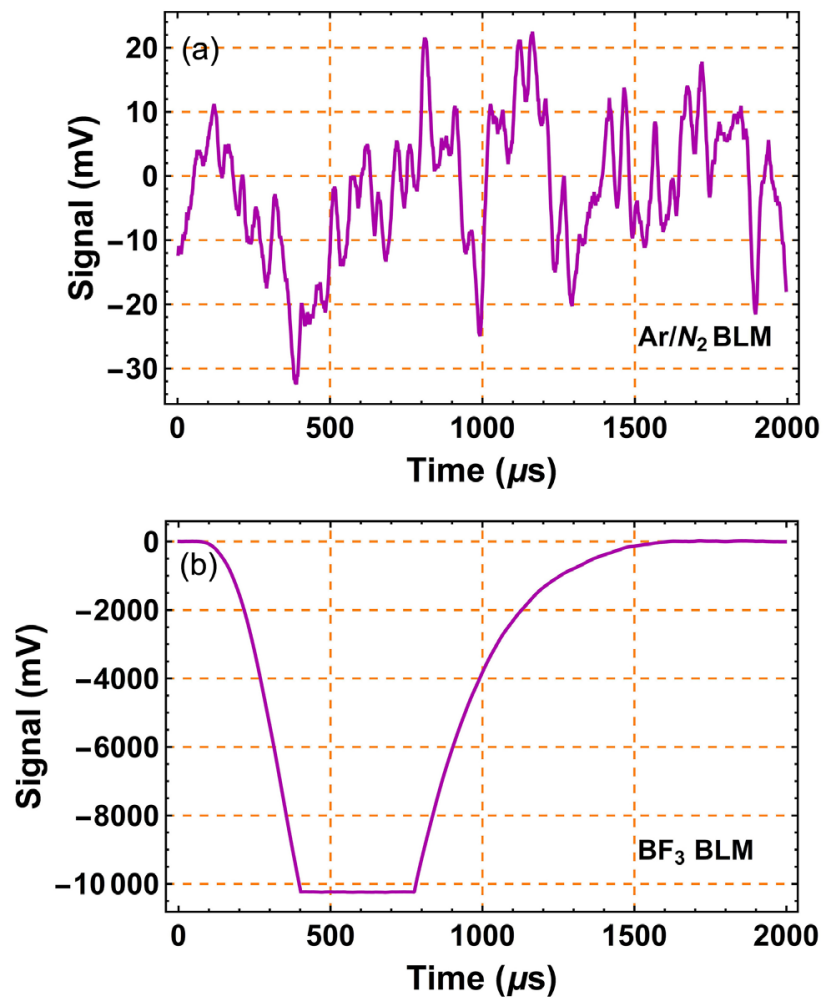

FIG. 27. The acquired waveforms of beam loss signal for the $\mathrm{BF}_{3}$ and $\mathrm{Ar} / \mathrm{N}_{2} \mathrm{BLM}$ in a same beam loss event.

$$
E_{\text {out }}=\frac{E}{n !}\left(\frac{t}{\tau}\right)^{n} e^{-t / \tau},
$$

where $E_{\text {out }}$ is the output voltage, $t$ the time, $n$ the number of integration stages, and the other variables are the fitting parameters. Figure 28 shows an unsaturated experimental waveform from another beam loss test and the fitting results with different $n$ values. It can be seen the fitting accords well with the waveform when $n$ equals 5 .

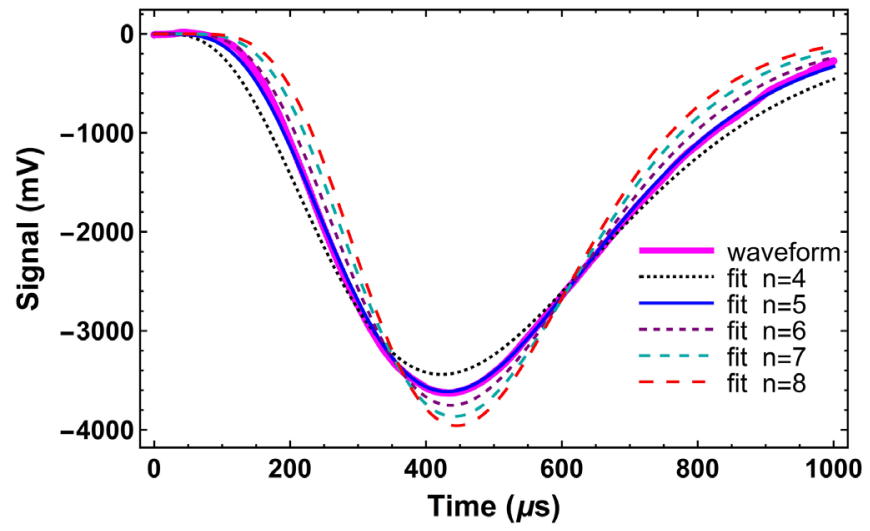

FIG. 28. The unsaturated experimental waveform of beam loss signal for $\mathrm{BF}_{3}$ monitor and the fitting results with different $n$ values.
Since the low sensitivity of $\mathrm{Ar} / \mathrm{N}_{2}$ BLM to beam loss at the beam energy of $15 \mathrm{MeV}$, the hardest issue is that $\mathrm{Ar} / \mathrm{N}_{2}$ BLM cannot generate an observable signal waveform. In order to validate the real ratio of beam loss signal for the two types of BLMs, we intentionally mismatch the magnet parameters in a single-shot mode to generate a beam-orbit distortion, which will create a relatively big loss. The waveforms in a loss event are shown in Fig. 29. Although this method can generate a valid signal for $\mathrm{Ar} / \mathrm{N}_{2} \mathrm{BLM}$, however, the number of lost particles still remains unknown, so the relative comparison method is adopted to study the signal contrast of the two monitors. The Ar/ $\mathrm{N}_{2}$ monitor eventually presents an observable beam loss signal, while the waveform of the $\mathrm{BF}_{3}$ monitor reaches an oversaturated level. Applying the framework of Eq. (11) to fit the waveforms, the saturation region of the $\mathrm{BF}_{3}$ signal could be complemented, and both fits agree well with the experimental data. The fitting amplitude is respectively 149.5 $( \pm 2.0) \mathrm{V}$ for the $\mathrm{BF}_{3}$ monitor and $91( \pm 0.87) \mathrm{mV}$ for the $\mathrm{Ar} / \mathrm{N}_{2}$ monitor. The signal of the $\mathrm{BF}_{3}$ monitor is about $1642( \pm 27)$ times higher than that of the $\mathrm{Ar} / \mathrm{N}_{2}$ monitor, which is approximately consistent with the FLUKA simulation $(\sim 1197)$ in the order of magnitude. The difference is caused by the uncertainty of the actual beam loss. In this
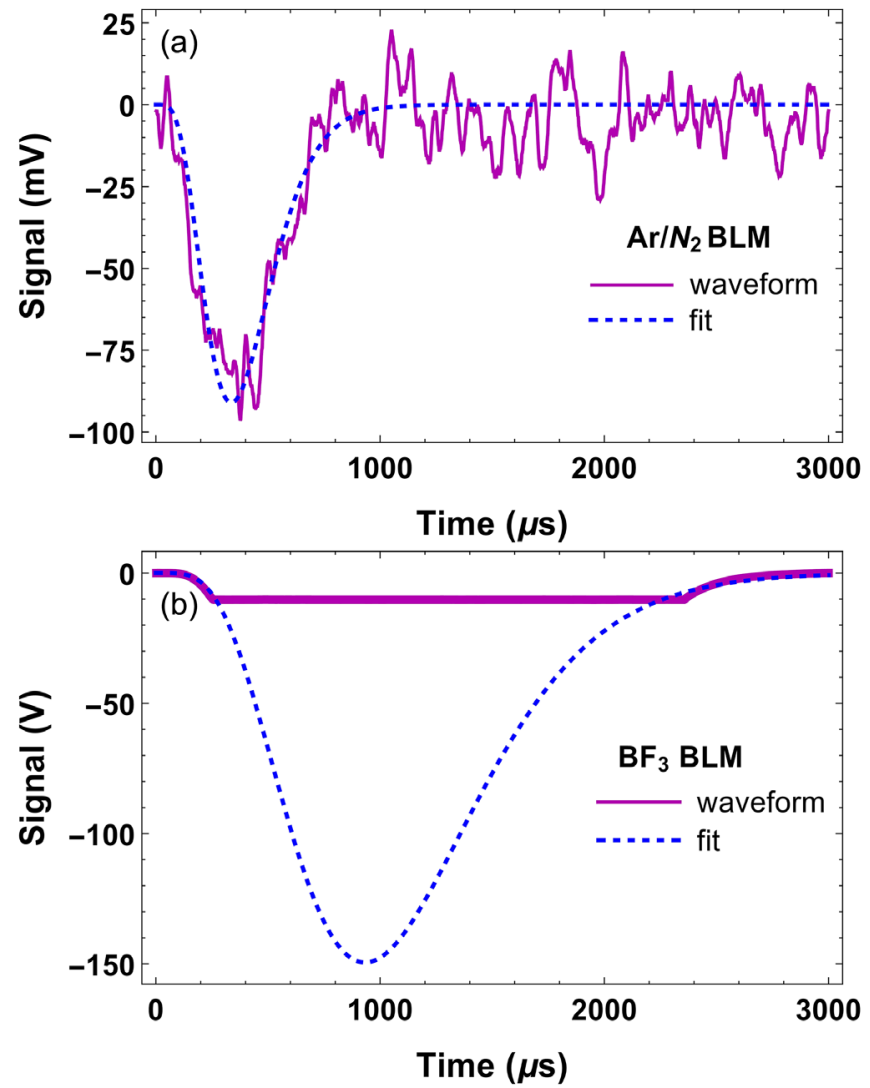

FIG. 29. The waveforms of the beam loss signal and the waveform fit in a controlled beam loss event for the (a) $\mathrm{Ar} / \mathrm{N}_{2} \mathrm{BLM}$ and (b) $\mathrm{BF}_{3}$ BLM. 
experiment, the beam loss is not entirely localized since several downstream BLMs not merely the two investigated ones observe the beam loss signal obviously larger than that in the normal machine operation. Besides, the beam energy is set to be monoenergetic $(15 \mathrm{MeV})$ in simulations, while particles may lose slightly forward or backward relative to the BLM location. In other words, the energy of lost particles would have a distribution rather than be monoenergetic. However, through detailed simulations and experiments, it demonstrates that the detection of thermal neutrons is an effective way to monitor the beam loss in the low energy section of a proton accelerator.

Unfortunately, everything goes both ways, the PE layer has a delay effect on the beam loss signal. The intrinsic delay time is about $80 \mu \mathrm{s}$ for the currently used $\mathrm{BF}_{3}$ monitor enclosed by a $7.5-\mathrm{cm}$ thick PE layer in this beam loss detection experiment. The time delay effect is caused by the thermalization and diffusion process of neutrons in the PE [54], and a long delay time is unfavorable for the machine protection, so the PE thickness should be optimized to balance the detection efficiency and the signal delay. Figure 30 illustrates a 2D histogram of neutron arriving in the $\mathrm{BF}_{3}$ monitor as a function of neutron time of flight (TOF) and neutron energy, and the simulated amount of lost primary particles is $10^{11}$. One can see a thermal neutron zone emerges in the phase space diagram as
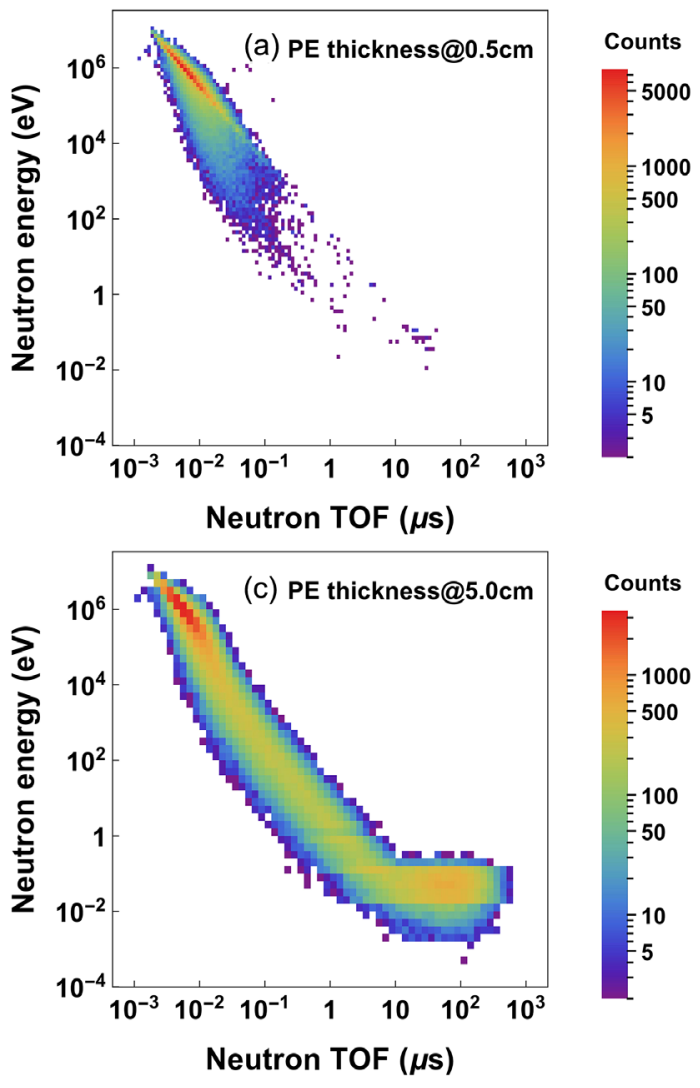

increasing the PE thickness, and the neutron TOF roughly ranges from $\sim$ ns to hundreds of $\mu$ s depending on the PE thickness. Similar experimental results of the time responses have been reported for the slow module of neutron sensitive BLM system in ESS, which also presents a maximum time delay of a few hundreds of $\mu$ s caused by the moderator layer [55]. Figure 31 presents the average neutron TOF and the corresponding energy deposition versus different PE thicknesses. In consideration of the wide range of reaction possibilities, the calculated average neutron TOF is weighted by the ${ }^{10} \mathrm{~B}$ capture cross section. The average neutron TOF increases as the PE thickness increases since the diffusion process needs more time and is about $99 \mu$ s for the $7.5-\mathrm{cm}$ thick PE, which is consistent with the real waveform at the beginning part $(\sim 80 \mu \mathrm{s})$ within an order of magnitude. The energy deposition increases and then reaches a plateau because there exist two competitive mechanisms to determine the final signal: the neutron thermalization and diffusion process, and the neutron shielding effect as increasing the PE thickness. When PE thickness exceeds $3.0 \mathrm{~cm}$, the energy deposition approximately reaches a plateau and even has a slight decrease tendency caused by the shielding effect for PE thickness greater than $6.0 \mathrm{~cm}$. Meanwhile, the average neutron TOF for 3.0-cm thick PE is about $32.7 \mu$ s, which presents considerable mitigation for the time-delay effect.

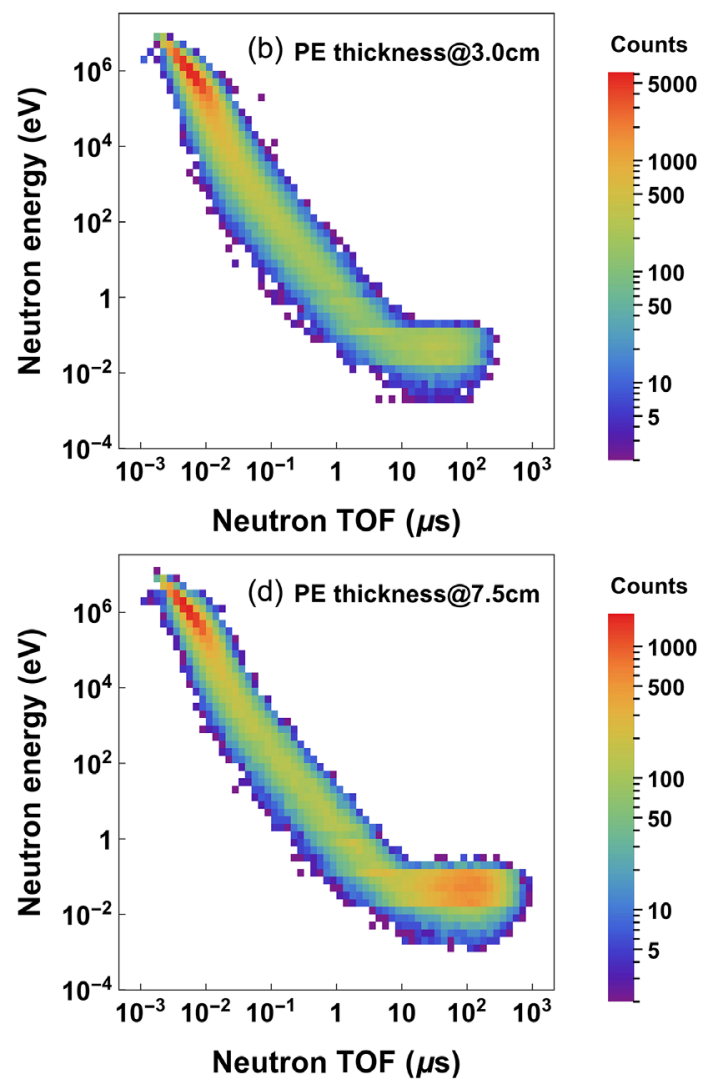

FIG. 30. The number of neutrons arriving in the $\mathrm{BF}_{3}$ monitor plotted versus the neutron TOF and energy in a $2 \mathrm{D}$ histogram. The amount of lost protons is $10^{11}$. 


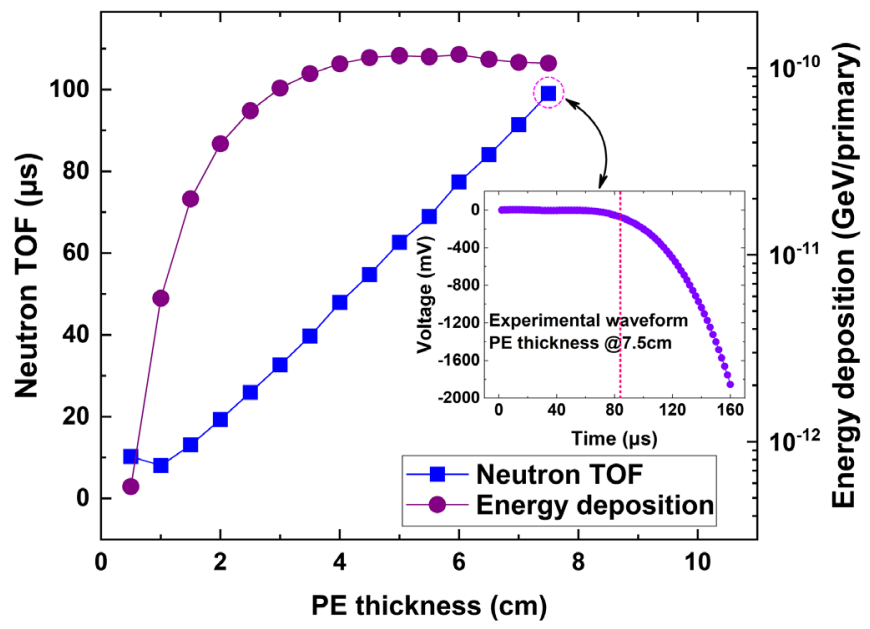

FIG. 31. The average neutron TOF and the energy deposition of $\mathrm{BF}_{3}$ monitor with different PE thickness.

The relevant experiments will be carried out to validate the simulations in the near future, and the beam loss detection for lower beam energy should also be researched.

Multiple scattering of neutrons will lead to considerable uncertainty for the loss location by the detection of neutrons. Therefore, the spatial resolution of the source position of beam loss should be concerned. This problem can be simplified and investigated by getting the distribution of secondary neutrons. A standard deviation of
0.9-3.6 $\mathrm{m}$ was reported for the secondary neutron distributions in ESS DTL, which was performed by GEANT4 simulations for a set of point loss scenarios [56,57]. Here our problem is treated in FLUKA by placing several planes at different positions out of the linac DTL to score the neutron hit positions. The planes are perpendicular to the $x$ axis ( $x=0$ corresponds to the centerline of the DTL tank) with a size of $10 \times 10 \mathrm{~m}^{2}$. The beam energy is set to be $15 \mathrm{MeV}$ and the loss position is set at $z=0$ with a loss angle of $1 \mathrm{mrad}$.

Figure 32 shows the hit maps of secondary neutrons induced by $10^{9}$ lost primaries and the neutron distribution along the $z$-axis direction. It can be seen from Fig. 32(a) that the kinetic energy of most neutrons is around the magnitude of $\mathrm{MeV}$. Neutrons spread wider on the YZ plane at $100 \mathrm{~cm}$ compared to $50 \mathrm{~cm}$, which implies that a better spatial resolution can be obtained for a neutron-sensitive BLM closer to the DTL tank. Figure 32(b) presents the onedimensional distribution of neutrons and the fit for it by projecting the 2D hit map on the $z$ axis. The full width at half maximum (FWHM) is adopted as the spatial resolution. It is seen that the distribution curve is almost Gaussian, however, Lorentzian distribution is used to fit the histogram because of the wider tails [58]. The spatial resolution is respectively 111.83 and $197.34 \mathrm{~cm}$ for the YZ plane at 50 and $100 \mathrm{~cm}$, which is comparable in magnitude with that in ESS DTL. The differences result from the different accelerator structures, beam parameters, and loss
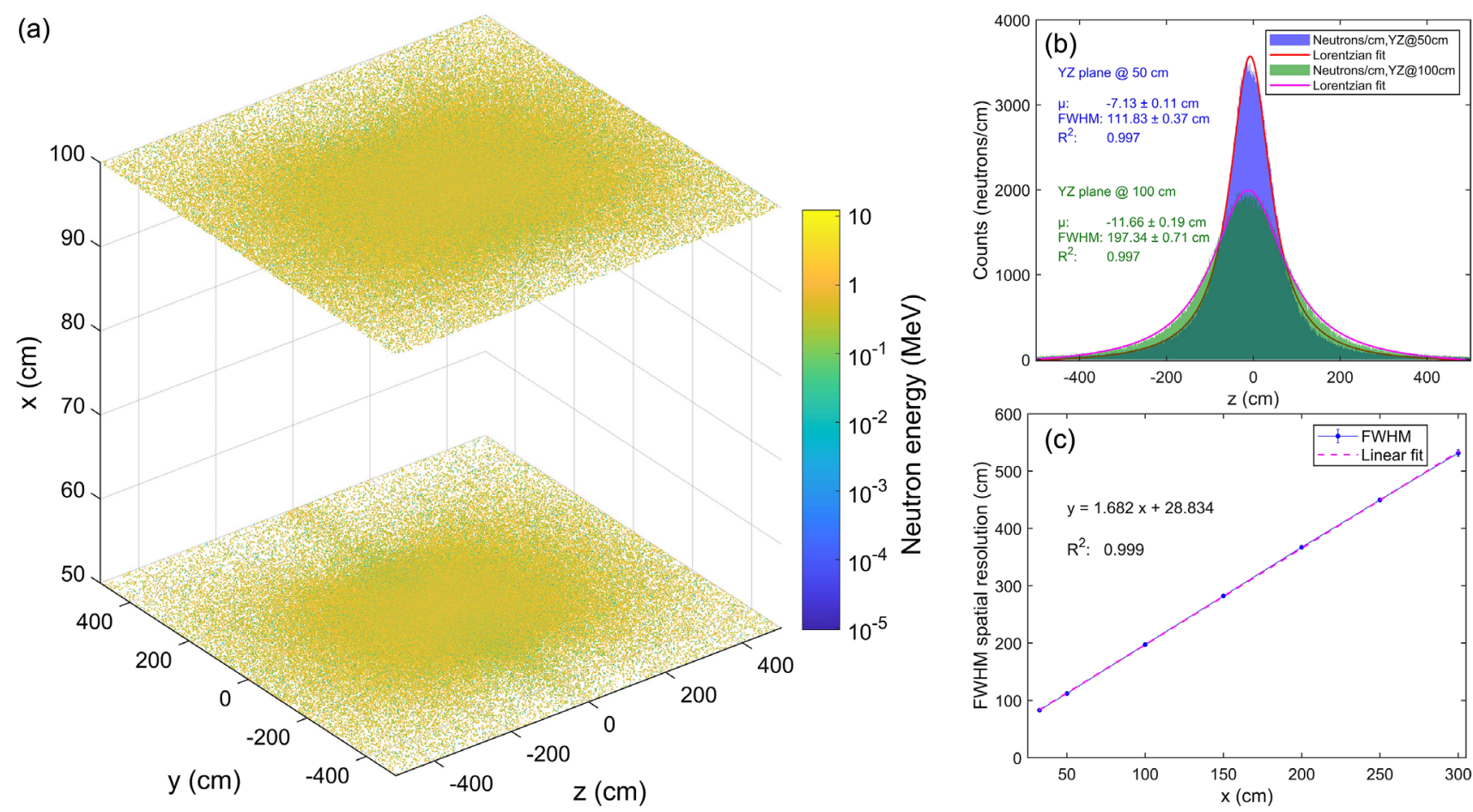

FIG. 32. (a) The neutron hit maps on the YZ plane respectively at 50 and $100 \mathrm{~cm}$ for the number of lost particles set as $10^{9}$, (b) the neutron one-dimensional distribution by projecting the $2 \mathrm{D}$ hit maps on the $\mathrm{z}$ axis, and (c) the spatial resolution of neutron distribution vs the positions of YZ plane. 
scenarios in simulations. Figure 32(c) reveals a linear relation of the spatial resolution with locations of the YZ plane. In our experimental configurations, as shown in Fig. 25(a), the simulated spatial resolution is $\sim 2 \mathrm{~m}$.

\section{CONCLUSION}

Beam loss detection is one of the most important issues in a proton accelerator. This paper presents detailed experimental and Monte Carlo studies for BLMs used in CSNS.

Response functions of CSNS BLMs are investigated at the incident angle of $0^{\circ}, 45^{\circ}, 180^{\circ}$, and $90^{\circ}$ for various secondary particles. The variation trends of response functions with the particle energy have been analyzed. The $\gamma$-ray calibration experiment was carried out to calibrate the BLMs and validate the correctness of the FLUKA program. The different dose rates in this experiment were controlled by the distance of BLM to the radiation source, and simulations show a good agreement with measurements. The beam loss measurement in a mixed radiation field was performed with a movable slit-type emittance monitor on the temporary test stand for beam diagnostics during the CSNS project construction. The lost beam current was measured by the CTs, and both experiment and simulation results show a roughly linear relation of the measured BLM current with the lost beam current.

Furthermore, we simulate the BLM responses in several assumed beam loss scenarios from the linac DTL to the RCS part in the CSNS, which gives a rough dynamic range of BLM and the signal tendency with the increase of beam energy. Based on the two-steps method, the different contributions of secondary particles to the total signal of BLM have been calculated. It reveals that the main contribution of the signal is from the photon and followed by the neutron in the linac DTL part. In the RCS part, the beam loss responses nearby a quadrupole magnet are sensitive to the BLM positions. The major contribution in our geometric configuration in the RCS part is given by the proton and followed by $\pi^{ \pm}$, neutron and photon. The $\mu^{ \pm}$ contribution is rather small and can be ignored in all scenarios. The ionization-current responses are obtained according to different loss rates and scenarios, and the space charge effects are also taken into account. The simulated current for scenarios with $0.1 \%$ local loss in the RCS part shows a wide dynamic range exceeding the currently used electronics, and a $10^{-5}$ local loss could be effectively detected in the RCS.

In addition, we perform a detailed study of the beam loss detection in the low energy section of the DTL part where the low fluences of secondary particles bring out the detection difficulty. The $\mathrm{BF}_{3}$ monitor was adopted to detect neutrons in the secondary radiation field. First, the beam loss responses were simulated and then the relevant experiments were carried out for the beam energy at $\sim 15 \mathrm{MeV}$ during the machine commissioning. Both simulation and experiment show the signal of the $\mathrm{BF}_{3}$ monitor is about 3 orders of magnitude higher than that of the normal $\mathrm{Ar} / \mathrm{N}_{2}$ monitor used in CSNS. The time delay caused by the neutron moderation is the main drawback for the method based on the thermal-neutron detection. Simulations give the neutron TOF and energy deposition for different PE thickness. The corresponding experiment work will be executed to validate the simulation in the near future, and the beam loss detection at lower energy will also be carried out. Finally, the FWHM spatial resolution for the neutronbased beam loss detection is estimated from the distribution of secondary neutrons. It shows a resolution of $\sim 2 \mathrm{~m}$ for our experimental configurations and a linear relation with the distance of BLM relative to the DTL tank.

\section{ACKNOWLEDGMENTS}

This work is supported by the National Natural Science Foundation of China (Grants No. 11575219, No. 11705215, and No. 11805220). The authors would like to thank our colleagues Hantao Jing, Qingbiao Wu, Wanju Luo from CSNS, and M. Polkovnikov from IHEP of Russian for their helpful discussions in the FLUKA simulations; thank colleagues Yuwen An, Liangsheng Huang, and Hanyang Liu for their discussions in the beam loss mechanisms and patterns; and thank colleagues Changjun Ning, Pengcheng Wang and Lei Liu for supplying some useful mechanical drawings. The authors also would like to thank Ms. Qian Xu for her help in the data processing.

[1] J. Wei et al., China Spallation Neutron Source: Design, R\&D, and outlook, Nucl. Instrum. Methods Phys. Res., Sect. A 600, 10 (2009).

[2] A. Lechner, C. Bracco, F. Cerutti, A. Christov, L. S. Esposito, N. V. Shetty, and V. Vlachoudis, Energy deposition studies for fast losses during LHC injection failures, in Proceedings of the 4th International Particle Accelerator Conference, IPAC-2013, Shanghai, China, 2013 (JACoW, Shanghai, China, 2013), TUPFI027, p. 1397.

[3] K. Wittenburg, Beam loss monitors, CERN Accelerator School: Course on Beam Diagnostics, Dourdan, France, 2008 [Report No. CERN-2009-005, Geneva, 2009], p. 249.

[4] A. Smolyakov, E. Mustafin, N. Pyka, and P. Spiller, Radiation damage studies for the slow extraction from SIS100, in Proceedings of the 11th European Particle Accelerator Conference, Genoa, 2008 (EPS-AG, Genoa, Italy, 2008), p. 3602.

[5] A. Lechner et al., Validation of energy deposition simulations for proton and heavy ion losses in the CERN Large Hadron Collider, Phys. Rev. Accel. Beams 22, 071003 (2019).

[6] E. B. Holzer et al., Beam loss monitoring system for the LHC, IEEE Nuclear Science Symposium Conference Record, 2005 (IEEE, Fajardo, PR, 2005), p. 1052.

[7] S. Lee, M. Tanaka, T. Toyama, and J. Kishiro, The beam loss monitor system of the J-PARC linac, $3 \mathrm{GeV}$ RCS and 
$50 \mathrm{GeV} \mathrm{MR}$, in the 9th European Particle Accelerator Conference, EPAC-2004, Lucerne, Switzerland, 2004 (JACoW, Lucerne, Switzerland, 2004), p. 2667.

[8] Z. Liu, J. Crisp, T. Russo, R. Webber, and Y. Zhang, A new beam loss detector for low-energy proton and heavy-ion accelerators, Nucl. Instrum. Methods Phys. Res., Sect. A 767 (2014).

[9] M. C. Ross and D. McCormick, A coaxial cable beam loss monitor ion chamber system for high power multibunch beams, Report No. SLAC-PUB-7944, 1998.

[10] E. Janata, Determination of location and intensity of radiation through detection of Cherenkov emission in optical fibers: Part 1. Method and experimental, Nucl. Instrum. Methods Phys. Res., Sect. A 493, 1 (2002).

[11] X. M. Maréal, Y. Asano, and T. Itoga, Design, development, and operation of a fiber-based Cherenkov beam loss monitor at the SPring-8 Angstrom Compact Free Electron Laser, Nucl. Instrum. Methods Phys. Res., Sect. A 673, 32 (2012).

[12] D. Gassner, P. Cameron, C. Mi, and R. Witkover, Spallation Neutron Source beam loss monitor system, in Proceedings of the 2003 Particle Accelerator Conference, Portland, OR (IEEE, New York, 2003), p. 2447.

[13] L. Tchelidze, H. Hassanzadegan, M. Jarosz, and A. Jansson, Beam Loss Monitoring at the European Spallation Source, in the 2nd International Beam Instrumentation Conference, IBIC2013, Oxford, UK, 2013 (JACoW, Oxford, UK, 2013), p. 795.

[14] R. L. Witkover, E. Zitvogel, and R. Michnoff, RHIC beam loss monitor system design, in Proceedings of the Particle Accelerator Conference, Vancouver, BC, Canada, 1997 (IEEE, New York, 1997), p. 2218.

[15] G. F. Knoll, Radiation Detection and Measurement, 4th ed. (John Wiley \& Sons, Inc., New York, 2010).

[16] K. Wittenburg, Beam loss monitoring and control, in Proceedings of the 8th European Particle Accelerator Conference, Paris, 2002 (EPS-IGA and CERN, Geneva, 2002), p. 109.

[17] A.P. Zhukov, Beam loss monitors (BLMs): Physics, simulations and applications in accelerators, in Proceedings of the 14th Beam Instrumentation Workshop, Santa Fe, NM (LANL, Los Alamos, 2010), THTNB01, p. 553.

[18] A. Ferrari, P. Sala, A. Fassò, and J. Ranft, FluKA: A multiparticle transport code, Technical Reports No. CERN2005-10, No. INFN/TC_05/11, No. SLAC-R-773, and No. CERN/INFN, 2005.

[19] T. T. Böhlen, F. Cerutti, M. P. W. Chin, A. Fassò, A. Ferrari, P. G. Ortega, A. Mairani, P. R. Sala, G. Smirnov, and V. Vlachoudis, The FLUKA Code: Developments and challenges for high energy and medical applications, Nucl. Data Sheets 120, 211 (2014).

[20] G. Battistoni et al., Overview of the FluKa code, Ann. Nucl. Energy 82, 10 (2015).

[21] A. Fontana et al., 3rd FLUKA Advanced Course and Workshop (LNF, Frascati, Rome, Italy, 2014).

[22] E. Lebbos, Measurements and simulations of the BLM response to a radiation field inside the CERF target area, Report No. CERN-EN-NOTE-2010-002-STI, 2010.

[23] K. S. Krane, Introductory Nuclear Physics (John Wiley \& Sons, Inc., New York, 1987).
[24] V. Vlachoudis, FLAIR: a powerful but user friendly graphical interface for FLUKA, in Proceedings of the International Conference on Mathematics, Computational Methods \& Reactor Physics, M\&C 2009, Saratoga Springs, New York, 2009 (American Nuclear Society, LaGrange Park, IL, 2009).

[25] L. Landau, On the energy loss of fast particles by ionization, J. Phys. (Moscow) 8, 201 (1944).

[26] W. R. Leo, Techniques for Nuclear and Particle Physics Experiments (Springer-Verlag, Berlin 1994).

[27] F.H. Attix, Introduction to Radiological Physics and Radiation Dosimetry (Wiley-VCH, Weinheim, 1986).

[28] C. Zankowski and E. B. Podgorsak, Determination of saturation charge and collection efficiency for ionization chambers in continuous beams, Med. Phys. 25, 908 (1998).

[29] K. R. Kase, B. E. Bjärngard, and F. H. Attix, The Dosimetry Of Ionizing Radiation (Academic Press, New York, 1987), Vol. II.

[30] R. B. Zwaska, Ph.D. thesis, University of Texas at Austin, 2005.

[31] M. A. Biondi and L. M. Chanin, Blanc's law-ion mobilities in helium-neon mixtures, Phys. Rev. 122, 843 (1961).

[32] F. Sauli, Gaseous Radiation Detectors (Cambridge University Press Cambridge, England 2014).

[33] B. Dehning et al., LHC beam loss detector design: Simulation and measurements, in Proceedings of the 22nd Particle Accelerator Conference, PAC-2007, Albuquerque, NM (IEEE, New York, 2007), p. 4198.

[34] M. Brugger, E. Lebbos, M. Sapinski, and M. Stockner, Response functions of ionization chamber beam loss monitor, Technical Report No. EDMS ID: 1055210, CERN, Geneva, Switzerland, 2010.

[35] M. Stockner, B. Dehning, C. Fabjan, G. Ferioli, and E. B. Holzer, Measurements and Simulations of Ionization Chamber Signals in Mixed Radiation Fields for the LHC BLM System, Proceedings, 2006 IEEE Nuclear Science Symposium and Medical Imaging Conference NSS/MIC 2006, San Diego, CA, US, 2006 (IEEE, San Diego, 2006), p. 1342.

[36] M. Stockner, Ph.D. thesis, Vienna University of Technology (Report No. CERN-THESIS-2008-099, 2007).

[37] B. J. Allen, A. R. de L. Musgrove, J. W. Boldeman, M. J. Kenny, and R. L. Macklin, Resonance neutron capture in ${ }^{56} \mathrm{Fe}$, Nucl. Phys. A269, 408 (1976).

[38] R. L. Macklin, Neutron capture in the $1.15-\mathrm{keV}$ resonance of iron, Nucl. Sci. Eng. 83, 309 (1983).

[39] B. Buyuk, Gamma attenuation behavior of some stainless and boron steels, Acta Phys. Pol. A 127, 1342 (2015).

[40] T. Yang et al., Thermomechanical studies for the slit in the emittance measurements, J. Nucl. Sci. Technol. 53, 727 (2016).

[41] A. Lechner, Particle interactions with matter, CERN Yellow Reports: School Proceedings, Report No. CERN2018-008-SP 2018 (CERN, Geneva, 2018), p. 47.

[42] I. Strašík, E. Mustafin, and M. Pavlovic, Residual activity induced by heavy ions and beam-loss criteria for heavyion accelerators, Phys. Rev. Accel. Beams 13, 071004 (2010).

[43] Q. B. Wu, Q. B. Wang, J. M. Wu, and Z. J. Ma, Study on induced radioactivity of China Spallation Neutron Source, Chin. Phys. C 35, 596 (2011). 
[44] N. V. Mokhov and W. Chou, in the 7th ICFA Mini-Workshop on High Intensity High Brightness Hadron Beams: Beam Halo and Scraping, Lake Como, Wisconsin, 1999 [Report No. FERMILAB-CONF-00-185, 2000].

[45] L. Tchelidze, Beam loss limits in high power proton linear accelerators, in Proceedings of the 4th International Particle Accelerator Conference, IPAC-2013, Shanghai, China, 2013 (Ref. [2]), p. 3930.

[46] M. Cauchi, R. W. Assmann, A. Bertarelli, F. Carra, L. Lari, and A. Rossi, Thermomechanical assessment of the effects of a jaw-beam angle during beam impact on Large Hadron Collider collimators, Phys. Rev. Accel. Beams 18, 021001 (2015).

[47] Y. Nie, R. Schmidt, V. Chetvertkova, G. Rosell-Tarragó, F. Burkart, and D. Wollmann, Numerical simulations of energy deposition caused by $50 \mathrm{MeV}-50 \mathrm{TeV}$ proton beams in copper and graphite targets, Phys. Rev. Accel. Beams 20, 081001 (2017).

[48] I. D. Kittelmann and T. Shea, Simulations and detector technologies for the beam loss monitoring system at the ESS linac, in Proceedings, in the 57th ICFA Advanced Beam Dynamics Workshop on High-Intensity and HighBrightness Hadron Beams, HB2016, Malmö, Sweden, 2016 (JACoW, Malmö, Sweden, 2016), p. 553.

[49] I. L. Azhgirey, I. S. Bayshev, I. A. Kurochkin, V. A. Pikalov, O. V. Sumaneev, and V.S. Lukanin, Neutron monitors for high energy accelerators, in the 26th Russian Particle Accelerator Conference (RUPAC2018), Protvino, Russia, 2018 (JACoW, Protvino, Russia, 2018), p. 224.
[50] S. Assadi and A. Zhukov, Beam-loss measurement and simulation of low-energy SNS linac, in Proceedings of the 23rd International Linac Conference, LINAC-2006, Knoxville, TN, 2006 (JACoW, Knoxville, TN, 2006), p. 202.

[51] S. F. Mughabghab, Atlas of Neutron Resonances. Resonance Parameters and Thermal Cross Sections, $Z=1-100$, 5th ed. (Elsevier Science, New York, 2006).

[52] R. T. Kouzes, A. T. Lintereur, and E. R. Siciliano, Progress in alternative neutron detection to address the helium-3 shortage, Nucl. Instrum. Methods Phys. Res., Sect. A 784, 172 (2015).

[53] The official ENDF site, https://www.nndc.bnl.gov.

[54] D. G. Cacuci, Handbook of Nuclear Engineering (Springer, Boston, 2010).

[55] L. Segui et al., A micromegas based neutron detector for the ESS beam loss monitoring, in the 7th International Beam Instrumentation Conference, IBIC2018, Shanghai, China, 2018 (JACoW, Shanghai, China, 2018), p. 211.

[56] I. D. Kittelmann, Report regarding the MC simulations for the BLM-focus on the nBLM, ESS Technical Report No. ESS-0066428, 2016.

[57] I. D. Kittelmann et al., Neutron sensitive beam loss monitoring system for the ESS linac, in the 8th International Beam Instrumentation Conference, IBIC2019, Malmö, Sweden, 2019 (JACoW, Malmö, Sweden, 2019), p. 131.

[58] O. Behnke, K. Kröninger, G. Schott, and T. S. Sadenius, Data Analysis in High Energy Physics (Wiley-VCH, New York, 2013). 